\title{
Article \\ Patterns of Psychological Responses among the Public during the Early Phase of COVID-19: A Cross-Regional Analysis
}

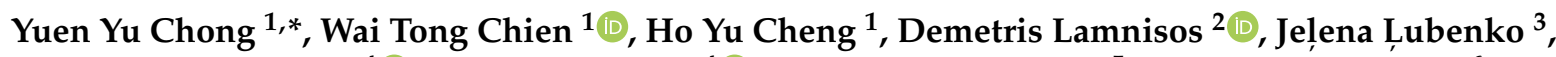 \\ Giovambattista Presti ${ }^{4}\left(\mathbb{D}\right.$, Valeria Squatrito ${ }^{4}{ }^{D}$, Marios Constantinou ${ }^{5}$, Christiana Nicolaou ${ }^{6}$, \\ Savvas Papacostas ${ }^{7}{ }^{\circledR}$, Gökçen Aydin ${ }^{8}{ }^{\circ}$, Francisco J. Ruiz ${ }^{9}{ }^{\circledR}$, Maria B. Garcia-Martin ${ }^{10}$, \\ Diana P. Obando-Posada ${ }^{10}$, Miguel A. Segura-Vargas ${ }^{9}$, Vasilis S. Vasiliou ${ }^{11}{ }^{(0}$, Louise McHugh ${ }^{12}{ }^{\mathbb{D}}$,

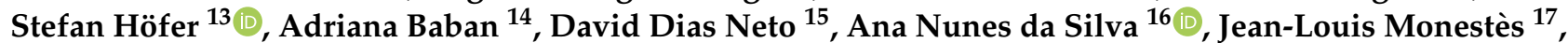 \\ Javier Alvarez-Galvez ${ }^{18}{ }^{\circledR}$, Marisa Paez Blarrina ${ }^{19}$, Francisco Montesinos ${ }^{20}$, Sonsoles Valdivia Salas ${ }^{21}{ }^{1}$, \\ Dorottya Óri ${ }^{22}{ }^{(D}$, Bartosz Kleszcz ${ }^{23}$, Raimo Lappalainen ${ }^{24}$, Iva Ivanović ${ }^{25}$, David Gosar ${ }^{26}{ }^{\circledR}$, Frederick Dionne ${ }^{27}$, \\ Rhonda M. Merwin ${ }^{28}$, Andrew T. Gloster ${ }^{29}$, Maria Karekla ${ }^{30}{ }^{1}$ and Angelos P. Kassianos ${ }^{30,31} \mathbb{C}$
}

check for updates

Citation: Chong, Y.Y.; Chien, W.T.; Cheng, H.Y.; Lamnisos, D.; L,ubenko, J.; Presti, G.; Squatrito, V.; Constantinou, M.; Nicolaou, C.; Papacostas, S.; et al. Patterns of Psychological Responses among the Public during the Early Phase of COVID-19: A Cross-Regional Analysis. Int. J. Environ. Res. Public Health 2021, 18, 4143. https:// doi.org/10.3390/ijerph18084143

Academic Editors: Andrea Fiorillo and Maurizio Pompili

Received: 23 March 2021

Accepted: 8 April 2021

Published: 14 April 2021

Publisher's Note: MDPI stays neutra with regard to jurisdictional claims in published maps and institutional affiliations.

Copyright: (c) 2021 by the authors. Licensee MDPI, Basel, Switzerland. This article is an open access article distributed under the terms and conditions of the Creative Commons Attribution (CC BY) license (https:// creativecommons.org/licenses/by/ $4.0 /)$
1 The Nethersole School of Nursing, Faculty of Medicine, The Chinese University of Hong Kong, Hong Kong, China; wtchien@cuhk.edu.hk (W.T.C.); hycheng@cuhk.edu.hk (H.Y.C.)

2 Department of Health Sciences, European University Cyprus, 1516 Nicosia, Cyprus; D.Lamnisos@euc.ac.cy

3 Psychological Laboratory, Faculty of Public Health and Social Welfare, Riga Stradinš University, LV-1007 Riga, Latvia; jelena.lubenko@rsu.lv

4 Kore University Behavioral Lab (KUBeLab), Faculty of Human and Social Sciences, Kore University of Enna, 94100 Enna, Italy; nanni.presti@gmail.com (G.P.); valeria.squatrito@gmail.com (V.S.)

5 Department of Social Sciences, School of Humanities and Social Sciences, University of Nicosia, 2417 Nicosia, Cyprus; constantinou.m@unic.ac.cy

6 Department of Nursing, Cyprus University of Technology, 3036 Limassol, Cyprus; c.nicolaou@cut.ac.cy

7 Cyprus Institute of Neurology and Genetics, 1683 Nicosia, Cyprus; savvas@cing.ac.cy

8 Department of Psychological Counseling and Guidance, Faculty of Education, Hasan Kalyoncu University, Gaziantep 27010, Turkey; gokcenaydn@gmail.com

9 Department of Psychology, Fundación Universitaria Konrad Lorenz, Bogotà 110231, Colombia; franciscoj.ruizji@gmail.com (F.J.R.); diana.obando@unisabana.edu.co (M.A.S.-V.)

10 Faculty of Psychology, University of La Sabana, Chía 53753, Colombia; pcairns@ehealthinnovation.org (M.B.G.-M.); mariabelengarciamartin2@gmail.com (D.P.O.-P.)

11 School of Applied Psychology, University College Cork, T12 YN60 Cork, Ireland; v.vasiliou@ucc.ie

12 School of Psychology, University College Dublin, D04 V1W8 Dublin, Ireland; louise.mchugh@ucd.ie

13 Department of Medical Psychology, Innsbruck Medical University, 6020 Innsbruck, Austria; stefan.hoefer@i-med.ac.at

14 Department of Psychology, Babeş-Bolyai University (UBB), 400095 Cluj-Napoca, Romania; adrianababan@psychology.ro

15 ISPA-Instituto Universitário, APPsyCI-Applied Psychology Research Center Capabilities \& Inclusion, 1149-041 Lisbon, Portugal; d.neto@campus.ul.pt

16 Faculdade de Psicologia, Alameda da Universidade, Universidade de Lisboa, 1649-013 Lisbon, Portugal; anacatarinans@gmail.com

17 LIP/PC2S Lab, Univ. Grenoble Alpes, 38040 Grenoble, France; jlmonestes@yahoo.fr

18 Department of Biomedicine, Biotechnology and Public Health, University of Cadiz, 11003 Cadiz, Spain; javieralvarezgalvez@gmail.com

19 Instituto ACT, 28036 Madrid, Spain; marisa.paez@institutoact.es

20 Department of Psychology, European University of Madrid, 28670 Madrid, Spain; francisco.montesinos2011@gmail.com

21 Department of Psychology and Sociology, University of Zaragoza, 50009 Zaragoza, Spain; sonsoval@unizar.es

22 Heim Pal National Pediatric Institute, Department of Mental Health, 1089 Budapest, Hungary; oridorottya@gmail.com

23 Bartosz Kleszcz Psychotherapy and Training, ul. Aleja Zwycięstwa 31/8, 41-200 Sosnowiec, Poland; bkleszcz@gmail.com

24 Department of Psychology, University of Jyväskylä, FI-40014 Jyväskylä, Finland; raimo.i.lappalainen@jyu.fi

25 Clinic for Psychiatry, Clinical Center of Montenegro, 81110 Podgorica, Montenegro; ivanovicivaa@gmail.com

26 Department of Child, Adolescent and Developmental Neurology, University Children's Hospital,

University Medical Center, 1000 Ljubljana, Slovenia; davidgosar@yahoo.com

27 Département de Psychologie, Université du Québec à Trois-Rivières, Trois-Rivières, QC G9A 5H7, Canada; frederickdionne.psy@gmail.com 
28 Department of Psychiatry and Behavioral Science, Duke University, Durham, NC 27708, USA; rhonda.merwin@duke.edu

29 Division of Clinical Psychology and Intervention Science, University of Basel, 4001 Basel, Switzerland; andrew.gloster@unibas.ch

30 Department of Psychology, University of Cyprus, 1678 Nicosia, Cyprus; mkarekla@ucy.ac.cy (M.K.); angelos.kassianos@ucl.ac.uk (A.P.K.)

31 Department of Applied Health Research, University College London, London WC1E 6BT, UK

* Correspondence: conniechong@cuhk.edu.hk

\begin{abstract}
This study aimed to compare the mediation of psychological flexibility, prosociality and coping in the impacts of illness perceptions toward COVID-19 on mental health among seven regions. Convenience sampled online survey was conducted between April and June 2020 from 9130 citizens in 21 countries. Illness perceptions toward COVID-19, psychological flexibility, prosociality, coping and mental health, socio-demographics, lockdown-related variables and COVID-19 status were assessed. Results showed that psychological flexibility was the only significant mediator in the relationship between illness perceptions toward COVID-19 and mental health across all regions (all $p \mathrm{~s}=0.001-0.021$ ). Seeking social support was the significant mediator across subgroups (all $p \mathrm{~s}$ range $=<0.001-0.005)$ except from the Hong Kong sample $(p=0.06)$ and the North and South American sample $(p=0.53)$. No mediation was found for problem-solving (except from the Northern European sample, $p=0.009)$. Prosociality was the significant mediator in the Hong Kong sample $(p=0.016)$ and the Eastern European sample $(p=0.008)$. These findings indicate that fostering psychological flexibility may help to mitigate the adverse mental impacts of COVID-19 across regions. Roles of seeking social support, problem-solving and prosociality vary across regions.
\end{abstract}

Keywords: COVID-19; psychological flexibility; mental health; prosociality; survey

\title{
1. Introduction
}

The rapid spread of coronavirus 2019 (COVID-19) caused by SARS-Co-V-2 amplified by forced quarantine and national lockdowns across countries have been shown to impose profound impacts on public mental health. Several meta-analyses have highlighted that at least one-third of the populations worldwide have reported symptoms of depression, anxiety, stress, and insomnia during the early stage of the COVID-19 pandemic [1,2]. In contrast, these symptoms are more severe among people with pre-existing mental health problems [3], quarantined persons [2], COVID-19 patients [2,4], and health care professionals [5-7]. It is expected that the adverse mental health implications arising from the pandemic can be more prevalent and persistent than the infection itself, which deserves timely and joined global efforts for efficient and effective interventions.

With the shift of the epicenter from Mainland China to the United States and Europe, considerable variations in managing the COVID-19 pandemic have been found across the globe. For example, in the United States, the existing public health federalism allows flexibility for the State government officials to customize pandemic responses in accordance to the unique characteristics of state populations [8]. However, this creates complications in centralizing and coordinating manpower and resources across the states for implementing timely responses to address the pandemic [9]. In contrast, countries with similar infection trends such as Germany, Austria and Switzerland, who share similar federalism systems, were able to leverage state resources to implement protective policies efficiently [10]. There is a growing public consensus in implementing and adhering to a series of COVID19 precautionary measures such as physical distancing, event restrictions, temperature checking, and closures of schools and non-essential business [11]. In contrast, the wearing of face coverings has been less widely accepted and adopted in Europe than in Asia [12]. Apart from the aforementioned variations in COVID-19 restrictions, the pre-existing socioeconomic characteristics and the capacity of health care services of each individual country may have exacerbated existing health disparities across the globe $[11,13]$. In view of this 
complexity, it is expected that people across countries and regions may have different psychological responses when facing COVID-19, implying the plausible variations of strategies in addressing mental health.

In our previous work, we followed hypotheses derived from the Leventhal's Common Sense Model of Self-Regulation [14,15] to examine the mediating roles of coping, psychological flexibility (i.e., the capacity of being open to difficult experiences and committed toward values-driven goals [16]) and prosociality (i.e., attitudes and/or behaviors that are intended to help and benefit others [17]) in the impacts of illness perceptions toward COVID-19 on mental health [18] (see Figure 1). Using cross-sectional survey data of 514 Hong Kong adults, we found that other than those known coping factors (i.e., seeking social support, problem-solving, avoidance and positive thinking), psychological flexibility and prosociality were the two higher-order response styles that significantly mediated the impacts [18]. The purpose of the present study was to extend this single-site study evidence by investigating whether people in multiple worldwide regions exhibited similar coping patterns as of the Hong Kong sample. More specifically, in line with the theoretical bases derived from the Common Sense Model of Self-Regulation [14,15], we aimed to examine whether psychological flexibility and prosociality remained the fundamental aspects of protecting mental health among people across various geographical regions in the midst of COVID-19. In literature, only a few multi-country or multi-regional surveys have been conducted to document the prevalence of mental health illnesses in general public during the COVID-19 outbreak [19-22]. One recent meta-analysis of 55 surveys with a total of 189,159 participants has indicated that the prevalence of depression (16.2\%, 34 studies) and anxiety $(13.5 \%, 33$ studies) in studies conducted in China were similar to that of studies conducted in other Asian and European countries [5] (for depression: 16.9\%, 12 studies; for anxiety: $19.0 \%, 18$ studies). However, research that focuses on comparing the psychological responses and coping patterns of people in different countries or regions when facing the pandemic is currently lacking.

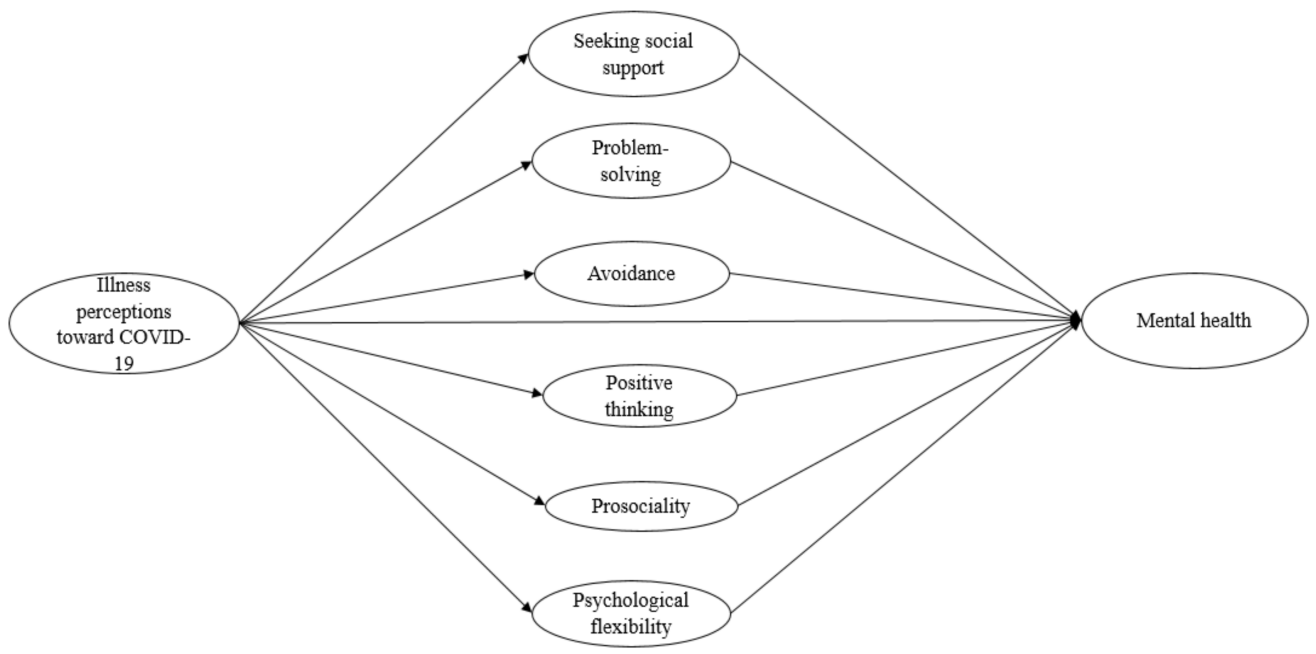

Figure 1. Hypothetical model of the study.

\section{Materials and Methods}

\subsection{Study Design and Participants}

The study was an online, multi-language cross-sectional survey, COVID-19 IMPACT (see https:/ / ucy.ac.cy/acthealthy/en/covid-19-impact-survey; accessed on 10 April 2020); its methodology of participant recruitment and data collection has been reported in our previous publications $[18,23,24]$. In brief, a total of 9565 individuals aged 18 years or above in 78 countries worldwide were conveniently recruited during the early phase of the COVID-19 pandemic (April to June 2020) through local press (e.g., newspapers, newsletters and radio stations), social media platforms, professional groups' email lists 
and networks, as well as the participating universities' mass emailing. These participants should be able to read at least one of the following languages (i.e., English, Greek, German, French, Spanish, Turkish, Dutch, Latvian, Italian, Portuguese, Finnish, Slovenian, Polish, Romanian, Chinese, Hungarian, Montenegrin, \& Persia), and access to internet services for completing the online survey. Participants who self-selected and enrolled in the study were invited to provide informed consent and completed a 20-min online survey via a secured Google platform.

\subsection{Measures}

The participants completed a battery of measures using the language of their choice:

1. the Mental Health Continuum Short Form for Adults (MHC-SF) assessing one's mental health focusing on emotional, social and psychological well-being (14 items, 6-point Likert scale) $[25,26]$;

2. the Brief Illness Perception Questionnaire (IPQ) items assessing the perceived consequences ("How much does COVID-19 affects your life?"), timeline ("How long do you think COVID-19 will continue?"), concern ("How much does COVID-19 worry you?") and emotional responses toward COVID-19 ("How much does the pandemic COVID-19 affect you emotionally (e.g., makes you sad, angry, scared, worried")? (4 items, 10-point Likert scale) [27];

3. the measures assessing the perceived susceptibility (3 items, 6-point Likert scale) and severity of COVID-19 (3 items, 6-point Likert scale) in line with the principles of the Health Belief Model [28];

4. the Brief Coping Orientation to Problems Experienced (Brief COPE) inventory composing of 28 items assessing a total of 14 coping strategies, which could be consolidated into four coping dimensions: seeking social support (venting, use of emotional support, use of instrumental support, religious belief); problem-solving (active coping, planning); avoidance (behavioral disengagement, self-distraction, substance use, denial, self-blaming) and positive thinking (humor, positive reframing, acceptance) [29-31];

5. the PsyFlex assessing all the six processes of psychological flexibility, including contacting the present moment, defusion, acceptance, self-as-context, values and committed action, of an individual (6 items, 5-point Likert scale) [32,33];

6. the Prosocialness Scale evaluating the level of prosocial behaviors, including sharing, helping, taking care of, and feeling empathic with others, which were carried out by the participant during the COVID-19 pandemic (6 items, 5-point Likert scale) [34].

The details of scoring instructions and psychometric properties of the aforementioned measures have been reported in our previous publications $[18,23,24]$. In summary, these measures showed satisfactory internal consistencies across participating regions (Cronbach's alphas $=0.76-0.85$ ) and adequate construct validity to their corresponding validation measures ( $r s=0.68-0.82)[25,29,33,34]$. In addition, the participants responded to questions about their sociodemographic characteristics, including their age, gender, country of residence, marital status, educational attainment, employment status, working as health care professionals (yes/no), and living status (living alone/others). Other COVID-19 related measures, such as the impact of lockdown on daily activities and financial situations, as well as the COVID-19 infection status of the participants (and their family members) were also assessed. The stringency of the COVID-19 precautionary measures per each participating country was tracked daily by the COVID-19 Government Response Tracker (OxCGRT). The OxCGRT was developed by the research team in the University of Oxford, which systematically summarized how the government responded in the following aspects: containment and closure such as restrictions in movement and closure of public areas (8 indicators), economic response (4 indicators) and health system polices (5 indicators) [35]. The Government Stringency Index score would then be calculated and rescaled in a range of 0 to 100, with the higher score indicating more stringent measures [35]. 


\subsection{Statistical Analysis}

We followed the recommendations given by the Population Division of the Department of Economic and Social Affairs of the United Nation to classify the participating countries into the following seven geographical regions: Eastern Asia, Western Asia, Northern and Southern America, Northern Europe, Western Europe, Southern Europe, and Eastern Europe [36]. Descriptive analyses and analysis of variance (ANOVA) tests were conducted to examine any significant differences on main study variables across the aforementioned regions (i.e., subgroups). The main analysis of this study consisted of testing a multiple-group structural equation model (SEM) using the SPSS AMOS version 23.0 (IBM Corp., Chicago, IL, USA) in which this model was hypothesized to illustrate the plausible mediating roles of four latent coping factors derived from the Brief COPE measure (i.e., seeking social support, problem-solving, avoidance, positive thinking), as well as the other two latent factors (i.e., prosociality and psychological flexibility) in the relationship between illness perceptions toward COVID-19 and mental health [18]. We firstly established the measurement models of all latent variables and then tested the hypothetical multiple mediation model for all the subgroups. The mediation effects were analyzed for the all the subgroups using bootstrapping method (5000 replications) with $95 \%$ bias-corrected confidence intervals. In addition, the chi-square difference test was employed to determine if there is any cross-group invariance when comparing two nested model, the unconstrained model in which no constraints were specified, and the constrained model wherein the parameters were constrained equal across the subgroups. The aforementioned SEM analyses were estimated by the maximum likelihood method, with the model fit indices (Comparative Fit Index (CFI) $\geq 0.90$; Tucker-Lewis Index (TLI) $\geq 0.90$; standardized root means square residual (SRMR) $\leq 0.10$; and root mean square error approximation (RMSEA) $\leq 0.08$ ) indicating an acceptable model fit $[18,24]$. The SEM was adjusted for the following sociodemographic variables, including age, gender, educational level, employment status and working as health care professionals (yes/no). The Government Stringency Index scores of the participating countries generated by the OxCGRT Indicators across the survey period were included as another covariate in our analysis. All statistical tests were two-sided and a $p$-value $<0.05$ was considered statistically significant.

\section{Results}

\subsection{Descriptive Statistics}

Owing to the fact that the survey was conducted online, and invitations were via social media and connections, the response rates of all study regions were unavailable. Of the 9867 respondents who accessed the survey website, 9565 provided completed data (96.9\% completion rate; $88.1-100 \%$ per region). A total of 435 out of 9565 participants $(4.5 \%)$ were excluded from the analysis as their corresponding countries received less than 100 completed survey responses. Table 1 summarizes the characteristics of the remaining 9130 participants in 21 countries across seven regions. The participants were mainly females $(77.6 \%$, range $=70.5-84.9 \%$ per region $)$, middle-aged $(55 \%$, range $=43.0-64.1 \%$ per region $)$ and employed on a full-time basis ( $53.7 \%$, range $=42.2 \%$ to $63.1 \%$ per region). Less than one-fifth of the participants were health care professionals, except for those from Western Europe which composed of over $30 \%$. More than two-thirds of the participants per region attained at least tertiary level of education. When social distancing and isolation measures began between April and June 2020 during the survey period, $47.1 \%$ of the participants stayed at home, but only $20.6 \%$ of those from Western Europe adhered to the measures. Around one-third of the participants $(n=3053)$ reported that their financial situations have got worse. A total of 133 participants (1.5\%), 68 participants' partners $(0.8 \%)$ and 519 participants' significant others (5.7\%) were infected by COVID-19, respectively. Of note, the mean score of the OxCGRT Indicator in Eastern Asia (i.e., Hong Kong, mean = 59.34, $\mathrm{SD}=8.71$ ) was lower than that of other regions (mean range $=67.63-76.83, \mathrm{SD}$ range $=$ 7.83-14.42), indicating the implementation of COVID-19 precautionary measures by the Hong Kong government were relatively less stringent during the survey period. 
Table 1. Characteristics of the participants per region.

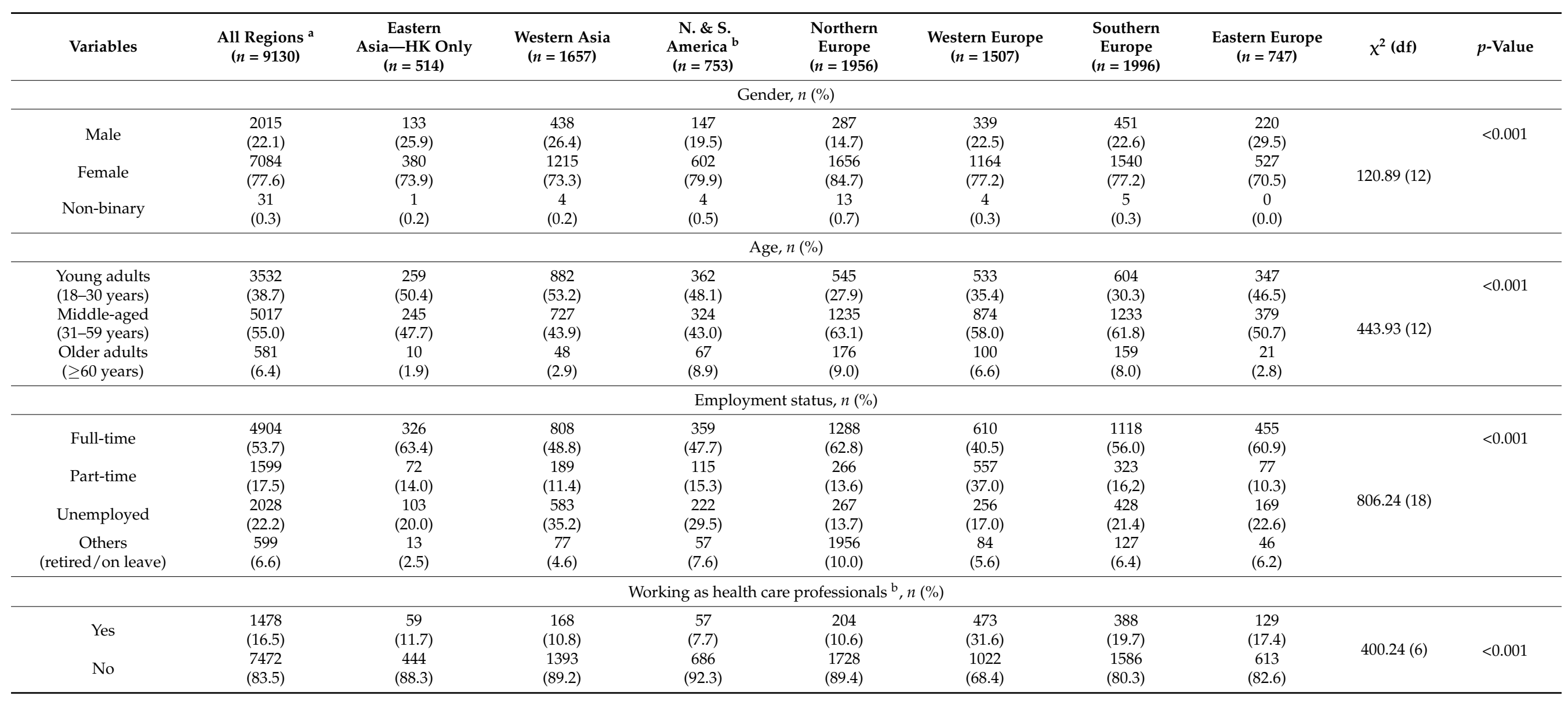


Table 1. Cont.

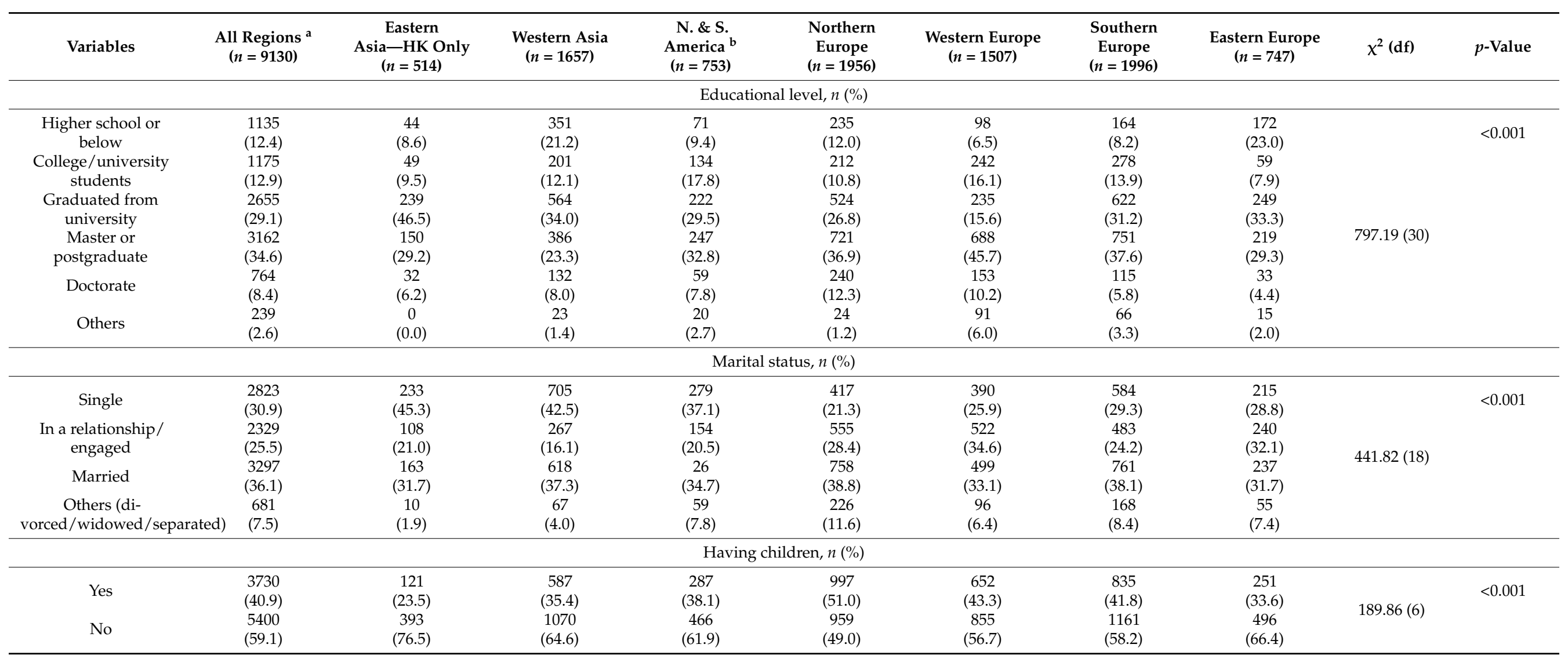


Table 1. Cont.

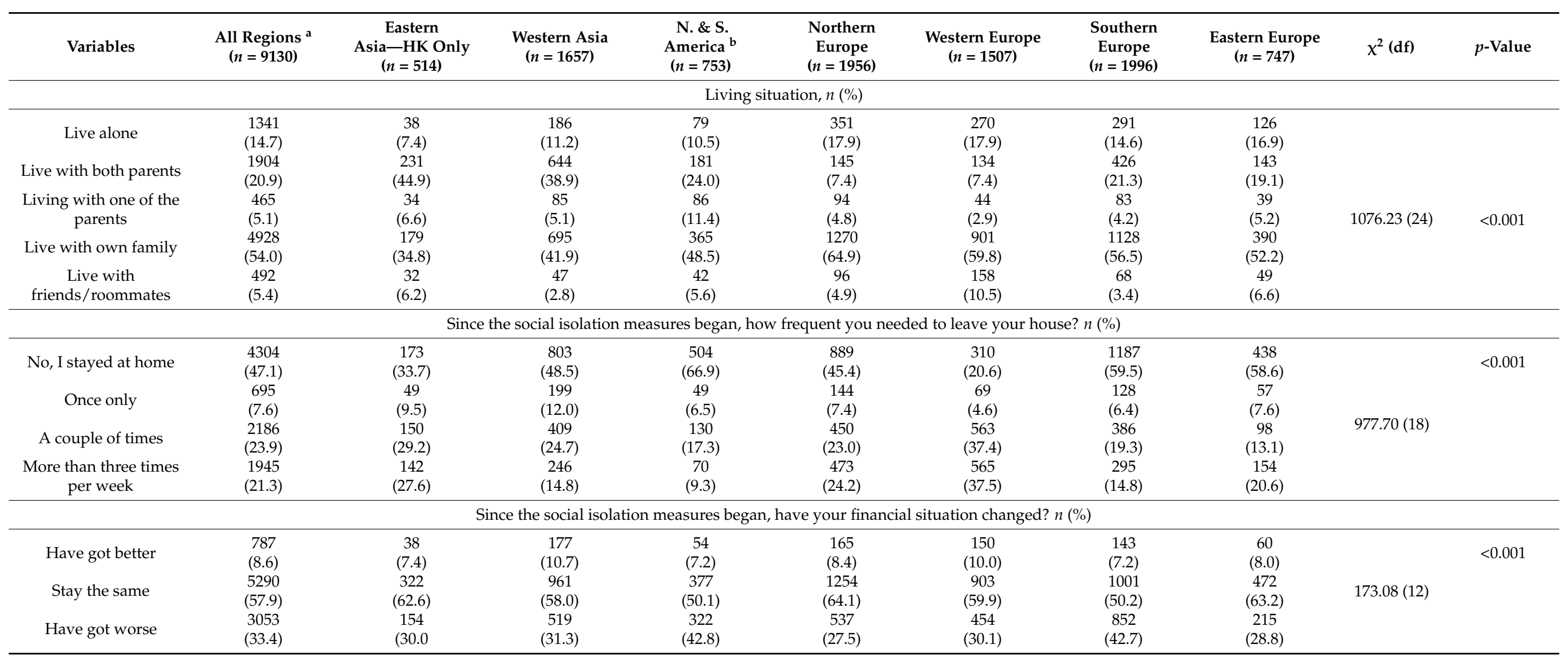


Table 1. Cont

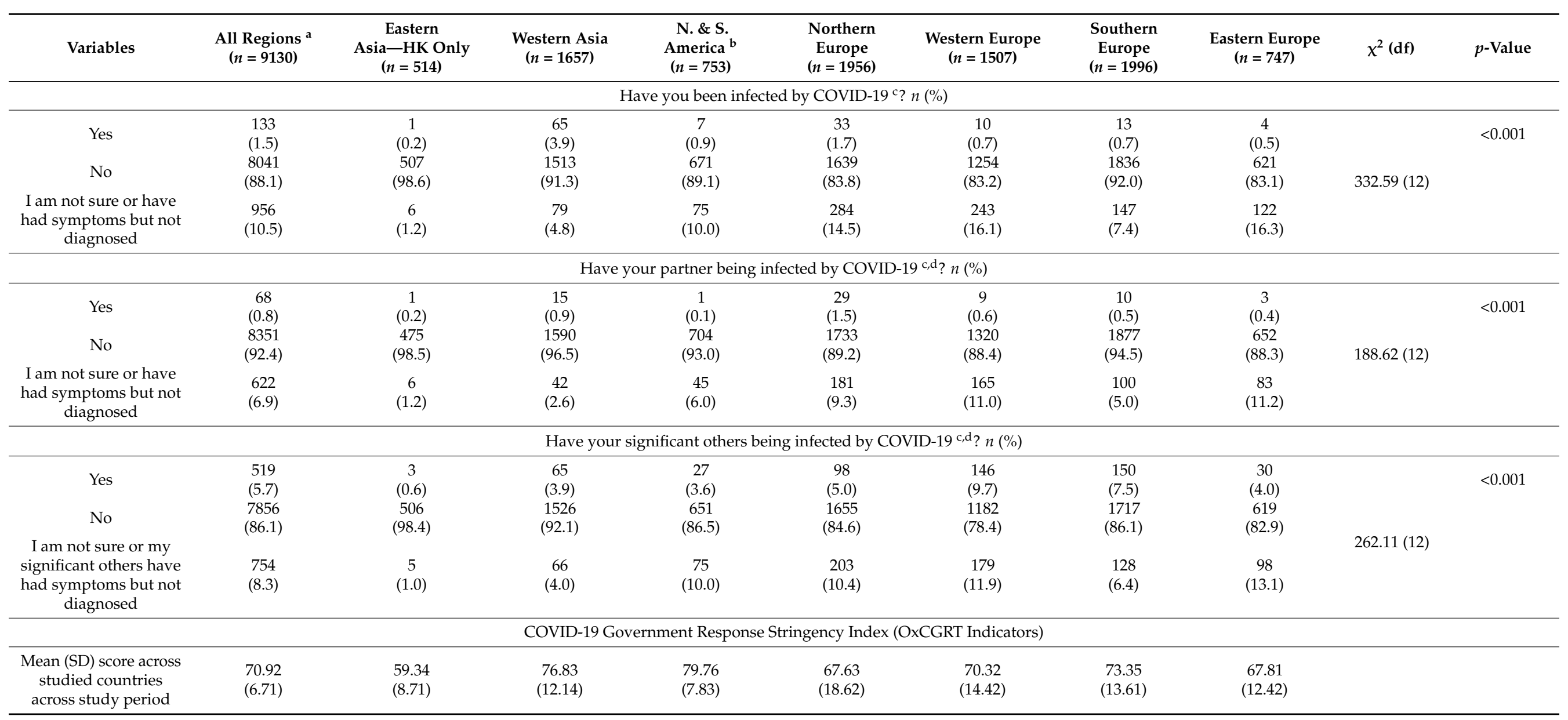

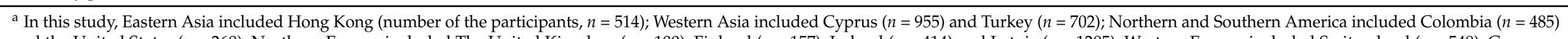

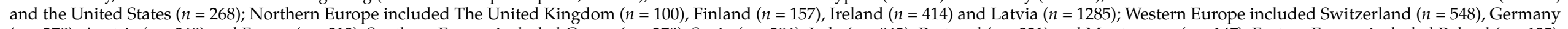

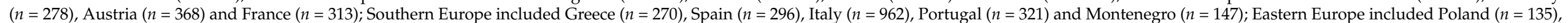

Romania $(n=339)$ and Hungary $(n=273) .{ }^{\mathrm{b}}$ N. \& S. America: Northern and Southern America. ${ }^{\mathrm{c}}$ Missing data $\leq 2 \% .{ }^{\mathrm{d}}$ COVID-19: Coronavirus 2019. 
Table 2 presents the illness perceptions toward COVID-19, coping, prosociality, psychological flexibility and mental health of the participants across regions. When compared with other geographical regions, analysis of variance followed by post-hoc comparisons indicated that the participants in the Eastern Asia region (i.e., Hong Kong people) reported the lowest scores in mental health (mean $=34.23, \mathrm{SD}=12.54$, mean difference [MD] range $=-4.12$ to -8.81 , all $p \mathrm{~s}<0.001$ ), psychological flexibility (mean $=19.43, \mathrm{SD}=4.02$, $\mathrm{MD}$ range $=-1.7$ to -3.4 , all $p \mathrm{~s}<0.001$ ) and prosociality (mean $=20.72, \mathrm{SD}=3.93, \mathrm{MD}$ range $=-2.12$ to -3.73 , all $p \mathrm{~s}<0.001$ ). In addition, Hong Kong people had stronger perceptions regarding the severity of COVID-19 when compared to those in other regions (mean $=14.55, \mathrm{SD}=3.02, \mathrm{MD}$ range $=0.99-3.43$, all $p \mathrm{~s}<0.001)$. Hong Kong people also attained the highest scores in behavioral disengagement (mean $=3.40, \mathrm{SD}=1.26$, MD range $=0.32$ to 0.84 , all $p \mathrm{~s}<0.001$ ) and self-blaming (mean $=3.46, \mathrm{SD}=1.45$, $\mathrm{MD}$ range $=0.24$ to 1.56 , all $p$ s range $=<0.001-0.001$ ), implying their tendencies in using maladaptive coping strategies, such as giving up to achieve goals and self-criticizing for things that happened, to manage their psychological difficulties. On the other hand, people in Western Asia reported the highest scores in active coping (mean $=6.11, \mathrm{SD}=1.45$, MD range $=0.33$ to 0.92 , all $p \mathrm{~s}<0.001$ ) and prosociality (mean $=24.38, \mathrm{SD}=3.94$, MD range $=0.52$ to 3.71 , all $p s<0.001$ ). The mental health scores across European regions (except those from Eastern Europe: mean $=37.18, \mathrm{SD}=14.09$ ) were generally similar (mean range $=41.29$ to $43.31, \mathrm{SD}$ range $=12.98$ to 14.09 ).

\subsection{Model Testing and Multiple-Group Structural Equation Model Analysis}

Similar to our previous reports $[18,24]$, the measuring items corresponding to the latent constructs were all adequately fit to the data representing the total sample. The hypothetical model was first tested among the total sample and demonstrated an acceptable fit to our data $\left(\chi^{2}=17927.22, d f=629, \mathrm{CFI}=0.92, \mathrm{TLI}=0.88\right.$, SRMR $=0.05$, RMSEA $\left.=0.04\right)$, supporting that this model could be retained for subsequent multiple-group SEM analysis. Chi-square difference test showed that there was a significant difference in the model fit between the constrained model (i.e., constraining all the structural parameters in the model to be equal across the seven subgroups) and the unconstrained model $\left(\Delta \chi^{2}=2188.75\right.$, $\Delta d f=258, p<0.001)$, indicating that the parameter coefficients differed significantly across the subgroups.

Table 3 shows the estimates of the direct and indirect effects regarding the interrelationships between illness perceptions toward COVID-19, coping and mental health based on the full unconstrained SEM model. The SEM model also showed an adequate fit to the data $\left(\chi^{2}=22386.96, d f=4403, \mathrm{CFI}=0.89, \mathrm{TLI}=0.85\right.$, SRMR $=0.05$, RMSEA $\left.=0.02\right)$. Psychological flexibility was the only factor that significantly mediated the relationship between illness perceptions toward COVID-19 and mental health across all subgroups ( $\beta$ range $=-0.15$ to -0.33 , SE range $=0.04$ to 0.12 , all $p$ s range $=0.001$ to 0.021 ). Seeking social support showed its significant mediating role across subgroups $(\beta$ range $=0.06-0.08$, SE range $=0.01$ to 0.03 , all $p$ s range $=<0.001$ to 0.005 ) except for the Hong Kong sample $(p=0.06)$ and the North and South American sample $(p=0.53)$. Similarly, avoidance also demonstrated its significant mediating role across subgroups $(\beta$ range $=-0.05$ to -0.32 , SE range $=0.03$ to 0.07 , all $p$ s range $=<0.001$ to 0.042) except for the Eastern Europe sample $(p=0.07)$. No mediated effect was found for problem-solving (except for the Northern Europe sample, $\beta=-0.04, \mathrm{SE}=0.01, p=0.009$ ). In the Hong Kong sample, prosociality $(\beta=0.05, \mathrm{SE}=0.01, p=0.016)$ and psychological flexibility $(\beta=-0.15, \mathrm{SE}=0.07, p=0.021)$ were the core mediators of protecting mental health. The Eastern Europe sample also showed similar coping patterns, but it additionally demonstrated seeking social support as the mediator $(\beta=0.08, \mathrm{SE}=0.03, p=0.005)$. For each subgroup SEM, the total variance explained by the predictors ranged from $56 \%$ to $73 \%$. 
Table 2. Illness perceptions toward COVID-19, coping, prosociality, psychological flexibility and mental health of the participants per region.

\begin{tabular}{|c|c|c|c|c|c|c|c|c|c|c|}
\hline & $\begin{array}{l}\text { All Regions }^{\mathrm{a}} \\
\quad(n=9130)\end{array}$ & $\begin{array}{c}\text { Eastern } \\
\text { Asia-HK Only } \\
(n=514)\end{array}$ & $\begin{array}{l}\text { Western Asia } \\
\quad(n=1657)\end{array}$ & $\begin{array}{c}\text { N. \& S. } \\
\text { America }{ }^{b} \\
(n=753)\end{array}$ & $\begin{array}{c}\text { Northern } \\
\text { Europe } \\
(n=1956)\end{array}$ & $\begin{array}{l}\text { Western Europe } \\
\quad(n=1507)\end{array}$ & $\begin{array}{c}\text { Southern } \\
\text { Europe } \\
(n=1996)\end{array}$ & $\begin{array}{l}\text { Eastern Europe } \\
\qquad(n=747)\end{array}$ & $F(d f)$ & $p$-Value \\
\hline $\begin{array}{c}\text { Variables } \\
\text { (Possible Range) }\end{array}$ & Mean (SD) & Mean (SD) & Mean (SD) & Mean (SD) & Mean (SD) & Mean (SD) & Mean (SD) & Mean (SD) & & \\
\hline \multicolumn{11}{|c|}{ Mental health } \\
\hline $\begin{array}{c}\text { Total score } \\
\quad(0-70)\end{array}$ & $\begin{array}{c}41.07 \\
(13.87)\end{array}$ & $\begin{array}{c}34.23 \\
(12.54)\end{array}$ & $\begin{array}{c}40.57 \\
(14.21)\end{array}$ & $\begin{array}{c}42.44 \\
(14.89)\end{array}$ & $\begin{array}{c}41.29 \\
(13.97)\end{array}$ & $\begin{array}{c}43.28 \\
(12.98)\end{array}$ & $\begin{array}{c}42.31 \\
(13.13)\end{array}$ & $\begin{array}{c}37.18 \\
(14.09)\end{array}$ & $42.47(6)$ & $<0.001$ \\
\hline $\begin{array}{c}\text { Emotional } \\
(0-15)\end{array}$ & $\begin{array}{l}10.15 \\
(3.34)\end{array}$ & $\begin{array}{c}8.73 \\
(3.06)\end{array}$ & $\begin{array}{c}9.60 \\
(3.50)\end{array}$ & $\begin{array}{l}10.57 \\
(3.43)\end{array}$ & $\begin{array}{l}10.42 \\
(3.24)\end{array}$ & $\begin{array}{l}10.94 \\
(3.06)\end{array}$ & $\begin{array}{l}10.23 \\
(3.25)\end{array}$ & $\begin{array}{c}9.46 \\
(3.51)\end{array}$ & $47.98(6)$ & $<0.001$ \\
\hline $\begin{array}{l}\text { Social } \\
(0-25)\end{array}$ & $\begin{array}{l}11.37 \\
(5.99)\end{array}$ & $\begin{array}{c}8.35 \\
(5.05)\end{array}$ & $\begin{array}{l}11.29 \\
(6.23)\end{array}$ & $\begin{array}{l}11.78 \\
(6.24)\end{array}$ & $\begin{array}{l}11.79 \\
(5.99)\end{array}$ & $\begin{array}{l}12.12 \\
(5.81)\end{array}$ & $\begin{array}{l}11.56 \\
(5.87)\end{array}$ & $\begin{array}{l}10.08 \\
(5.70)\end{array}$ & $34.86(6)$ & $<0.001$ \\
\hline $\begin{array}{c}\text { Psychological } \\
\quad(0-30)\end{array}$ & $\begin{array}{l}19.55 \\
(6.56)\end{array}$ & $\begin{array}{l}17.15 \\
(6.46)\end{array}$ & $\begin{array}{l}19.69 \\
(6.54)\end{array}$ & $\begin{array}{l}20.09 \\
(6.88) \\
\end{array}$ & $\begin{array}{l}19.08 \\
(6.81) \\
\end{array}$ & $\begin{array}{l}20.23 \\
(6.12)\end{array}$ & $\begin{array}{l}20.51 \\
(6.13)\end{array}$ & $\begin{array}{l}17.65 \\
(6.83)\end{array}$ & $35.24(6)$ & $<0.001$ \\
\hline \multicolumn{11}{|c|}{ Illness perceptions toward COVID-19 } \\
\hline $\begin{array}{c}\text { Consequence } \\
(1-10)\end{array}$ & $\begin{array}{c}7.40 \\
(2.24)\end{array}$ & $\begin{array}{c}6.84 \\
(1.88)\end{array}$ & $\begin{array}{c}7.96 \\
(2.12)\end{array}$ & $\begin{array}{c}7.25 \\
(2.17)\end{array}$ & $\begin{array}{c}7.48 \\
(2.21)\end{array}$ & $\begin{array}{c}6.49 \\
(2.36)\end{array}$ & $\begin{array}{c}7.83 \\
(2.07)\end{array}$ & $\begin{array}{c}7.15 \\
(2.37)\end{array}$ & $82.86(6)$ & $<0.001$ \\
\hline $\begin{array}{l}\text { Timeline } \\
(1-10)\end{array}$ & $\begin{array}{c}6.57 \\
(1.81)\end{array}$ & $\begin{array}{c}7.09 \\
(1.64)\end{array}$ & $\begin{array}{l}7.06 \\
(2.00)\end{array}$ & $\begin{array}{c}6.78 \\
(1.66)\end{array}$ & $\begin{array}{c}6.03 \\
(1.72)\end{array}$ & $\begin{array}{c}6.34 \\
(1.59)\end{array}$ & $\begin{array}{c}6.86 \\
(1.78)\end{array}$ & $\begin{array}{c}6.03 \\
(1.80)\end{array}$ & $85.57(6)$ & $<0.001$ \\
\hline $\begin{array}{l}\text { Concern } \\
(1-10)\end{array}$ & $\begin{array}{c}6.72 \\
(2.41)\end{array}$ & $\begin{array}{c}6.63 \\
(2.03)\end{array}$ & $\begin{array}{c}7.30 \\
(2.33)\end{array}$ & $\begin{array}{c}7.50 \\
(2.15)\end{array}$ & $\begin{array}{c}6.65 \\
(2.37)\end{array}$ & $\begin{array}{c}5.42 \\
(2.39)\end{array}$ & $\begin{array}{c}7.36 \\
(2.13)\end{array}$ & $\begin{array}{l}5.80 \\
(2.56)\end{array}$ & $160.04(6)$ & $<0.001$ \\
\hline $\begin{array}{l}\text { Emotional } \\
\text { responses }(1-10)\end{array}$ & $\begin{array}{c}6.39 \\
(2.51)\end{array}$ & $\begin{array}{c}6.28 \\
(2.09)\end{array}$ & $\begin{array}{c}6.97 \\
(2.51)\end{array}$ & $\begin{array}{c}6.84 \\
(2.45)\end{array}$ & $\begin{array}{c}6.38 \\
(2.53)\end{array}$ & $\begin{array}{c}5.64 \\
(2.49)\end{array}$ & $\begin{array}{c}6.49 \\
(2.41)\end{array}$ & $\begin{array}{c}5.99 \\
(2.60)\end{array}$ & $46.95(6)$ & $<0.001$ \\
\hline $\begin{array}{c}\text { Perceived } \\
\text { susceptibility } \\
(3-18)\end{array}$ & $\begin{array}{c}8.74 \\
(3.56)\end{array}$ & $\begin{array}{c}9.23 \\
(2.99)\end{array}$ & $\begin{array}{c}9.82 \\
(3.62)\end{array}$ & $\begin{array}{c}9.11 \\
(3.63)\end{array}$ & $\begin{array}{c}9.25 \\
(3.48)\end{array}$ & $\begin{array}{c}7.35 \\
(3.27)\end{array}$ & $\begin{array}{c}8.29 \\
(3.42)\end{array}$ & $\begin{array}{c}8.35 \\
(3.66)\end{array}$ & $85.04(6)$ & $<0.001$ \\
\hline $\begin{array}{c}\text { Perceived } \\
\text { severity (3-18) }\end{array}$ & $\begin{array}{l}12.42 \\
(3.70)\end{array}$ & $\begin{array}{l}14.55 \\
(3.02)\end{array}$ & $\begin{array}{l}11.90 \\
(3.94)\end{array}$ & $\begin{array}{l}13.56 \\
(3.52)\end{array}$ & $\begin{array}{l}12.83 \\
(3.54)\end{array}$ & $\begin{array}{l}11.12 \\
(3.28)\end{array}$ & $\begin{array}{l}12.86 \\
(3.64)\end{array}$ & $\begin{array}{l}11.37 \\
(3.77)\end{array}$ & $101.43(6)$ & $<0.001$ \\
\hline \multicolumn{11}{|c|}{ Seeking social support } \\
\hline $\begin{array}{c}\text { Venting } \\
(2-8)\end{array}$ & $\begin{array}{c}4.76 \\
(1.56)\end{array}$ & $\begin{array}{c}5.23 \\
(1.45)\end{array}$ & $\begin{array}{c}4.93 \\
(1.67)\end{array}$ & $\begin{array}{c}4.41 \\
(1.47)\end{array}$ & $\begin{array}{c}4.46 \\
(1.35)\end{array}$ & $\begin{array}{c}5.06 \\
(1.67)\end{array}$ & $\begin{array}{c}4.52 \\
(1.42)\end{array}$ & $\begin{array}{c}5.18 \\
(1.73)\end{array}$ & $57.49(6)$ & $<0.001$ \\
\hline $\begin{array}{l}\text { Use of emotional } \\
\text { support (2-8) }\end{array}$ & $\begin{array}{c}4.72 \\
(1.74)\end{array}$ & $\begin{array}{c}4.75 \\
(1.60)\end{array}$ & $\begin{array}{c}4.66 \\
(1.71)\end{array}$ & $\begin{array}{c}4.43 \\
(1.95)\end{array}$ & $\begin{array}{c}4.83 \\
(1.56)\end{array}$ & $\begin{array}{c}4.76 \\
(1.78)\end{array}$ & $\begin{array}{c}4.56 \\
(1.74)\end{array}$ & $\begin{array}{c}5.26 \\
(1.93)\end{array}$ & $20.66(6)$ & $<0.001$ \\
\hline $\begin{array}{l}\text { Use of } \\
\text { instrumental } \\
\text { support (2-8) }\end{array}$ & $\begin{array}{c}4.38 \\
(1.69)\end{array}$ & $\begin{array}{c}5.28 \\
(1.52)\end{array}$ & $\begin{array}{c}4.88 \\
(1.95)\end{array}$ & $\begin{array}{c}4.00 \\
(1.76)\end{array}$ & $\begin{array}{c}4.34 \\
(1.42)\end{array}$ & $\begin{array}{c}4.15 \\
(1.58)\end{array}$ & $\begin{array}{c}4.08 \\
(1.60)\end{array}$ & $\begin{array}{c}4.44 \\
(1.72)\end{array}$ & $73.93(6)$ & $<0.001$ \\
\hline $\begin{array}{l}\text { Religious belief } \\
\qquad(2-8)\end{array}$ & $\begin{array}{c}3.84 \\
(1.95)\end{array}$ & $\begin{array}{c}4.06 \\
(1.92)\end{array}$ & $\begin{array}{c}4.69 \\
(2.17)\end{array}$ & $\begin{array}{c}4.19 \\
(2.14)\end{array}$ & $\begin{array}{c}3.56 \\
(1.76)\end{array}$ & $\begin{array}{c}3.27 \\
(1.69)\end{array}$ & $\begin{array}{c}3.61 \\
(1.76)\end{array}$ & $\begin{array}{c}3.90 \\
(1.98)\end{array}$ & $96.39(6)$ & $<0.001$ \\
\hline
\end{tabular}


Table 2. Cont.

\begin{tabular}{|c|c|c|c|c|c|c|c|c|c|c|}
\hline & $\begin{array}{l}\text { All Regions }^{\mathrm{a}} \\
\quad(n=9130)\end{array}$ & $\begin{array}{c}\text { Eastern } \\
\text { Asia-HK Only } \\
(n=514)\end{array}$ & $\begin{array}{l}\text { Western Asia } \\
\quad(n=1657)\end{array}$ & $\begin{array}{c}\text { N. \& S. } \\
\text { America } \\
(n=753)\end{array}$ & $\begin{array}{c}\text { Northern } \\
\text { Europe } \\
(n=1956)\end{array}$ & $\begin{array}{l}\text { Western Europe } \\
\quad(n=1507)\end{array}$ & $\begin{array}{c}\text { Southern } \\
\text { Europe } \\
(n=1996)\end{array}$ & $\begin{array}{l}\text { Eastern Europe } \\
\quad(n=747)\end{array}$ & $F(d f)$ & $p$-Value \\
\hline $\begin{array}{c}\text { Variables } \\
\text { (Possible Range) }\end{array}$ & Mean (SD) & Mean (SD) & Mean (SD) & Mean (SD) & Mean (SD) & Mean (SD) & Mean (SD) & Mean (SD) & & \\
\hline \multicolumn{11}{|c|}{ Problem-solving } \\
\hline $\begin{array}{l}\text { Active coping } \\
(2-8)\end{array}$ & $\begin{array}{c}5.65 \\
(1.55)\end{array}$ & $\begin{array}{c}5.78 \\
(1.44)\end{array}$ & $\begin{array}{c}6.11 \\
(1.45)\end{array}$ & $\begin{array}{c}5.32 \\
(1.60)\end{array}$ & $\begin{array}{c}5.51 \\
(1.45)\end{array}$ & $\begin{array}{c}5.27 \\
(1.59)\end{array}$ & $\begin{array}{c}5.72 \\
(1.53)\end{array}$ & $\begin{array}{c}5.82 \\
(1.68)\end{array}$ & $52.84(6)$ & $<0.001$ \\
\hline $\begin{array}{l}\text { Planning } \\
(2-8)\end{array}$ & $\begin{array}{c}5.57 \\
(1.54)\end{array}$ & $\begin{array}{c}5.90 \\
(1.34)\end{array}$ & $\begin{array}{c}5.54 \\
(1.48)\end{array}$ & $\begin{array}{c}5.28 \\
(1.64)\end{array}$ & $\begin{array}{c}5.66 \\
(1.44)\end{array}$ & $\begin{array}{c}5.39 \\
(1.64)\end{array}$ & $\begin{array}{c}5.61 \\
(1.56)\end{array}$ & $\begin{array}{c}5.80 \\
(1.61)\end{array}$ & $16.53(6)$ & $<0.001$ \\
\hline \multicolumn{11}{|c|}{ Avoidance } \\
\hline $\begin{array}{c}\text { Behavioral } \\
\text { disengagement } \\
(2-8)\end{array}$ & $\begin{array}{c}2.86 \\
(1.23)\end{array}$ & $\begin{array}{c}3.40 \\
(1.26)\end{array}$ & $\begin{array}{c}2.99 \\
(1.33)\end{array}$ & $\begin{array}{c}2.73 \\
(1.25)\end{array}$ & $\begin{array}{c}3.06 \\
(1.24)\end{array}$ & $\begin{array}{c}2.62 \\
(1.13)\end{array}$ & $\begin{array}{c}2.69 \\
(1.10)\end{array}$ & $\begin{array}{c}2.73 \\
(1.21)\end{array}$ & $48.51(6)$ & $<0.001$ \\
\hline $\begin{array}{l}\text { Self-distraction } \\
\qquad(2-8)\end{array}$ & $\begin{array}{c}5.51 \\
(1.61)\end{array}$ & $\begin{array}{c}5.19 \\
(1.54)\end{array}$ & $\begin{array}{c}5.87 \\
(1.47)\end{array}$ & $\begin{array}{c}5.87 \\
(1.49)\end{array}$ & $\begin{array}{c}5.16 \\
(1.55)\end{array}$ & $\begin{array}{c}5.36 \\
(1.76)\end{array}$ & $\begin{array}{c}5.47 \\
(1.59)\end{array}$ & $\begin{array}{c}5.83 \\
(1.67)\end{array}$ & $47.65(6)$ & $<0.001$ \\
\hline $\begin{array}{l}\text { Substance use } \\
\qquad(2-8)\end{array}$ & $\begin{array}{c}2.60 \\
(1.22)\end{array}$ & $\begin{array}{c}2.69 \\
(1.34)\end{array}$ & $\begin{array}{c}2.37 \\
(1.00)\end{array}$ & $\begin{array}{c}2.66 \\
(1.41)\end{array}$ & $\begin{array}{c}2.82 \\
(1.26)\end{array}$ & $\begin{array}{c}2.64 \\
(1.24)\end{array}$ & $\begin{array}{c}2.37 \\
(0.97)\end{array}$ & $\begin{array}{c}3.00 \\
(1.58)\end{array}$ & $49.09(6)$ & $<0.001$ \\
\hline $\begin{array}{c}\text { Denial } \\
(2-8)\end{array}$ & $\begin{array}{c}2.93 \\
(1.32)\end{array}$ & $\begin{array}{c}2.84 \\
(1.16)\end{array}$ & $\begin{array}{c}3.83 \\
(1.50)\end{array}$ & $\begin{array}{l}2.60 \\
(1.14)\end{array}$ & $\begin{array}{c}2.81 \\
(1.15)\end{array}$ & $\begin{array}{c}2.45 \\
(0.95)\end{array}$ & $\begin{array}{c}2.83 \\
(1.25)\end{array}$ & $\begin{array}{c}2.91 \\
(1.44)\end{array}$ & $199.51(6)$ & $<0.001$ \\
\hline \multicolumn{11}{|c|}{ Positive thinking } \\
\hline Humor (2-8) & $\begin{array}{c}4.57 \\
(1.73)\end{array}$ & $\begin{array}{c}4.04 \\
(1.54)\end{array}$ & $\begin{array}{c}4.80 \\
(1.74)\end{array}$ & $\begin{array}{c}4.05 \\
(1.87)\end{array}$ & $\begin{array}{c}4.56 \\
(1.60)\end{array}$ & $\begin{array}{c}5.11 \\
(1.75)\end{array}$ & $\begin{array}{c}4.18 \\
(1.54)\end{array}$ & $\begin{array}{c}4.92 \\
(1.93)\end{array}$ & $73.36(6)$ & $<0.001$ \\
\hline $\begin{array}{l}\text { Positive reframing } \\
\qquad(2-8)\end{array}$ & $\begin{array}{c}5.78 \\
(1.62)\end{array}$ & $\begin{array}{c}5.50 \\
(1.45)\end{array}$ & $\begin{array}{l}6.15 \\
(1.56)\end{array}$ & $\begin{array}{c}5.31 \\
(1.73)\end{array}$ & $\begin{array}{c}5.68 \\
(1.52)\end{array}$ & $\begin{array}{c}6.10 \\
(1.62)\end{array}$ & $\begin{array}{c}5.53 \\
(1.63)\end{array}$ & $\begin{array}{c}5.93 \\
(1.64)\end{array}$ & $48.89(6)$ & $<0.001$ \\
\hline Acceptance (2-8) & $\begin{array}{c}6.55 \\
(1.35)\end{array}$ & $\begin{array}{c}6.17 \\
(1.34)\end{array}$ & $\begin{array}{c}6.17 \\
(1.34)\end{array}$ & $\begin{array}{c}6.51 \\
(1.37)\end{array}$ & $\begin{array}{c}6.72 \\
(1.13)\end{array}$ & $\begin{array}{c}6.85 \\
(1.36)\end{array}$ & $\begin{array}{c}6.61 \\
(1.27)\end{array}$ & $\begin{array}{c}6.90 \\
(1.36)\end{array}$ & $87.54(6)$ & $<0.001$ \\
\hline \multicolumn{11}{|c|}{ Psychological flexibility } \\
\hline Total score (6-30) & $\begin{array}{l}21.83 \\
(4.09)\end{array}$ & $\begin{array}{l}19.43 \\
(4.02)\end{array}$ & $\begin{array}{l}21.15 \\
(4.02)\end{array}$ & $\begin{array}{l}21.41 \\
(4.58)\end{array}$ & $\begin{array}{l}22.09 \\
(3.85)\end{array}$ & $\begin{array}{l}22.89 \\
(3.97)\end{array}$ & $\begin{array}{l}22.22 \\
(3.89)\end{array}$ & $\begin{array}{l}21.58 \\
(4.16)\end{array}$ & $62.57(6)$ & $<0.001$ \\
\hline \multicolumn{11}{|c|}{ Prosociality } \\
\hline Total score (6-30) & $\begin{array}{l}22.85 \\
(4.19)\end{array}$ & $\begin{array}{l}20.72 \\
(3.93)\end{array}$ & $\begin{array}{l}24.38 \\
(3.94)\end{array}$ & $\begin{array}{l}23.33 \\
(4.25)\end{array}$ & $\begin{array}{l}21.04 \\
(4.29)\end{array}$ & $\begin{array}{l}23.04 \\
(3.66)\end{array}$ & $\begin{array}{l}23.75 \\
(3.76)\end{array}$ & $\begin{array}{l}22.34 \\
(4.31)\end{array}$ & $152.61(6)$ & $<0.001$ \\
\hline
\end{tabular}

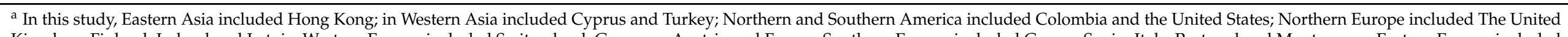

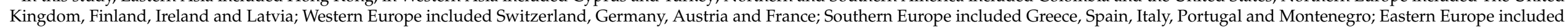
Poland, Romania and Hungary. ${ }^{b}$ N. \& S. America: Northern and Southern America. 
Table 3. Results of unconstrained multiple-group structural equation model by regions.

\begin{tabular}{|c|c|c|c|c|c|c|c|c|c|c|c|c|c|c|}
\hline \multirow[t]{2}{*}{$\begin{array}{c}\text { All } \\
\begin{array}{c}\text { Regions } \\
(n=9130)\end{array} \\
\end{array}$} & \multicolumn{2}{|c|}{$\begin{array}{l}\text { Eastern Asia, HK Only } \\
\qquad(n=514)\end{array}$} & \multicolumn{2}{|c|}{$\begin{array}{l}\text { Western Asia } \\
\quad(n=1657)\end{array}$} & \multicolumn{2}{|c|}{$\begin{array}{c}\text { N. \& S. } \\
\text { America } \\
(n=753)\end{array}$} & \multicolumn{2}{|c|}{$\begin{array}{c}\text { Northern } \\
\text { Europe } \\
(n=1956)\end{array}$} & \multicolumn{2}{|c|}{$\begin{array}{c}\text { Western } \\
\text { Europe } \\
(n=1507)\end{array}$} & \multicolumn{2}{|c|}{$\begin{array}{c}\text { Southern } \\
\text { Europe } \\
(n=1996)\end{array}$} & \multicolumn{2}{|c|}{$\begin{array}{c}\text { Eastern } \\
\text { Europe } \\
(n=747) \\
\end{array}$} \\
\hline & $B^{c}(\mathrm{SE})$ & $p$-Value & $\beta(\mathrm{SE})$ & $p$-Value & $\beta(\mathrm{SE})$ & $p$-value & $\beta$ (SE) & $p$-Value & $\stackrel{\beta}{\beta}$ & $p$-Value & $\beta$ (SE) & $p$-Value & $\begin{array}{l}\beta \\
\text { (SE) }\end{array}$ & $p$-Value \\
\hline \multicolumn{15}{|c|}{ Direct effects from illness perceptions toward COVID-19 (i.e., unstandardized path coefficient) } \\
\hline $\begin{array}{l}\text { Seeking social } \\
\text { support }\end{array}$ & $0.12(0.03)$ & $<0.001$ & $0.15(0.02)$ & $<0.001$ & $0.10(0.02)$ & $<0.001$ & $0.11(0.01)$ & $<0.001$ & $0.20(0.02)$ & $<0.001$ & $0.12(0.01)$ & $<0.001$ & $0.21(0.02)$ & $<0.001$ \\
\hline Problem-solving & $-0.04(0.03)$ & 0.22 & $0.02(0.02)$ & 0.24 & $0.09(0.02)$ & $<0.001$ & $0.04(0.01)$ & $<0.001$ & $0.10(0.02)$ & $<0.001$ & $0.12(0.01)$ & $<0.001$ & $0.11(0.03)$ & $<0.001$ \\
\hline Avoidance & $0.10(0.03)$ & $<0.001$ & $0.13(0.01)$ & $<0.001$ & $0.17(0.02)$ & $<0.001$ & $0.15(0.01)$ & $<0.001$ & $0.14(0.01)$ & $<0.001$ & $0.12(0.01)$ & $<0.001$ & $0.15(0.02)$ & $<0.001$ \\
\hline Positive thinking & $-0.02(0.01)$ & 0.19 & $-0.03(0.01)$ & $<0.001$ & $-0.03(0.01)$ & 0.01 & $-0.05(0.01)$ & $<0.001$ & $-0.11(0.01)$ & $<0.001$ & $-0.05(0.01)$ & $<0.001$ & $-0.02(0.01)$ & 0.033 \\
\hline $\mathrm{PF}^{\mathrm{c}}$ & $-0.12(0.02)$ & $<0.001$ & $-0.10(0.01)$ & $<0.001$ & $-0.14(0.02)$ & $<0.001$ & $-0.12(0.01)$ & $<0.001$ & $-0.09(0.01)$ & $<0.001$ & $-0.10(0.01)$ & $<0.001$ & $-0.13(0.02)$ & $<0.001$ \\
\hline \multicolumn{15}{|c|}{ Direct effects on mental health (i.e., unstandardized path coefficient) } \\
\hline $\begin{array}{l}\text { Seeking social } \\
\text { support }\end{array}$ & $0.19(0.16)$ & 0.043 & $0.41(0.12)$ & $<0.001$ & $-0.09(0.14)$ & 0.51 & $0.58(0.10)$ & $<0.001$ & $0.41(0.08)$ & $<0.001$ & $1.10(0.20)$ & $<0.001$ & $0.38(0.14)$ & 0.004 \\
\hline Problem-solving & $-0.57(.66)$ & 0.39 & $0.08(0.11)$ & 0.49 & $0.18(0.50)$ & 0.72 & $-0.84(0.28)$ & 0.008 & $-0.21(0.11)$ & 0.06 & $-0.40(0.29)$ & 0.17 & $-0.06(0.33)$ & 0.86 \\
\hline Avoidance & $-0.68(0.26)$ & 0.010 & $-0.41(0.20)$ & 0.041 & $-0.74(0.34)$ & 0.03 & $-0.72(0.25)$ & 0.003 & $\begin{array}{c}-10.02 \\
(0.34)\end{array}$ & 0.003 & $\begin{array}{c}-30.07 \\
(0.60)\end{array}$ & $<0.001$ & $-0.60(0.48)$ & 0.21 \\
\hline Positive thinking & $2.83(2.14)$ & 0.19 & $0.57(0.20)$ & 0.005 & $0.70(2.14)$ & 0.76 & $1.99(0.80)$ & 0.013 & $0.67(0.20)$ & $<0.001$ & $1.69(0.46)$ & $<0.001$ & $1.75(1.40)$ & 0.21 \\
\hline Prosociality & $0.48(0.22)$ & 0.029 & $.66(0.13)$ & $<0.001$ & $0.36(0.17)$ & 0.030 & $0.16(0.10)$ & 0.12 & $0.30(0.11)$ & 0.023 & $0.59(0.14)$ & $<0.001$ & $0.41(0.16)$ & 0.01 \\
\hline $\mathrm{PF}^{\mathrm{d}}$ & $1.30(0.28)$ & $<0.001$ & $2.43(0.18)$ & $<0.001$ & $2.35(0.38)$ & $<0.001$ & $2.45(0.26)$ & $<0.001$ & $2.53(0.26)$ & $<0.001$ & $1.15(0.35)$ & 0.001 & $2.58(0.29)$ & $<0.001$ \\
\hline $\mathrm{IP}^{\mathrm{e}}$ & $-0.09(0.06)$ & 0.13 & $-0.01(0.03)$ & 0.89 & $0.07(0.10)$ & 0.44 & $0.10(0.06)$ & 0.10 & $0.11(0.04)$ & 0.009 & $0.10(0.07)$ & 0.20 & $-0.04(0.07)$ & 0.55 \\
\hline \multicolumn{15}{|c|}{ Indirect effects } \\
\hline $\mathrm{IP} \rightarrow \mathrm{SS}^{\mathrm{f}} \rightarrow \mathrm{MH}^{\mathrm{g}}$ & $0.03(0.02)$ & 0.06 & $0.06(0.02)$ & 0.002 & $-0.01(0.01)$ & 0.53 & $0.07(0.01)$ & $<0.001$ & $0.07(0.02)$ & $<0.001$ & $0.12(0.03)$ & $<0.001$ & $0.08(0.03)$ & 0.005 \\
\hline $\mathrm{IP} \rightarrow \mathrm{PS}^{\mathrm{h}} \rightarrow \mathrm{MH}$ & $0.02(0.03)$ & 0.47 & $0.01(0.01)$ & 0.56 & $0.02(0.05)$ & 0.72 & $-0.04(0.01)$ & 0.009 & $-0.02(0.01)$ & 0.12 & $-0.08(0.03)$ & 0.16 & $-0.01(0.03)$ & 0.72 \\
\hline $\begin{aligned} \mathrm{IP} & \rightarrow \text { Avoidance } \\
\rightarrow \mathrm{MH} & \end{aligned}$ & $-0.07(0.03)$ & 0.04 & $-0.05(0.03)$ & 0.042 & $-0.13(0.06)$ & 0.035 & $-0.14(0.04)$ & 0.002 & $-0.12(0.04)$ & 0.004 & $-0.32(0.07)$ & $<0.001$ & $-0.13(0.07)$ & 0.07 \\
\hline $\mathrm{IP} \rightarrow \mathrm{PT}^{\mathrm{i}} \rightarrow \mathrm{MH}$ & $-0.06(0.05)$ & 0.30 & $-0.02(0.01)$ & 0.038 & $-0.01(0.06)$ & 0.75 & $-0.11(0.04)$ & 0.01 & $-0.07(0.03)$ & 0.015 & $-0.11(0.03)$ & 0.011 & $-0.05(0.03)$ & 0.14 \\
\hline $\begin{array}{c}\mathrm{IP} \rightarrow \text { Prosociaity } \\
\rightarrow \mathrm{MH}\end{array}$ & $0.05(0.01)$ & 0.016 & $-0.01(0.01)$ & 0.33 & $-0.01(0.01)$ & 0.08 & $0.00(0.00)$ & 0.39 & $0.00(0.00)$ & 0.39 & $-0.01(0.01)$ & 0.33 & $0.10(0.03)$ & 0.008 \\
\hline$\underset{\mathrm{MH}}{\mathrm{IP} \rightarrow \mathrm{PF}} \rightarrow$ & $-0.15(0.07)$ & 0.021 & $-0.24(0.03)$ & 0.002 & $-0.33(0.12)$ & 0.02 & $-0.29(0.05)$ & 0.002 & $-0.25(0.04)$ & 0.001 & $-0.16(0.05)$ & 0.008 & $-0.25(0.05)$ & 0.004 \\
\hline $\begin{array}{c}\text { Total } \\
\text { variance }\left(R^{2}\right)\end{array}$ & \multicolumn{2}{|c|}{0.64} & \multicolumn{2}{|c|}{0.56} & \multicolumn{2}{|c|}{0.67} & \multicolumn{2}{|c|}{0.72} & \multicolumn{2}{|c|}{0.66} & \multicolumn{2}{|c|}{0.73} & \multicolumn{2}{|c|}{0.72} \\
\hline
\end{tabular}

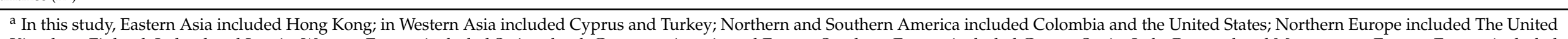

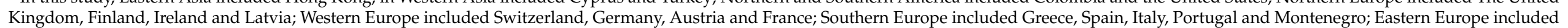

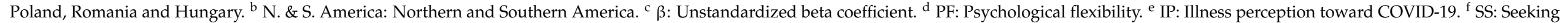
social support. ${ }^{\mathrm{g}} \mathrm{MH}$ : Mental health. ${ }^{\mathrm{h}}$ PS: Problem-solving. ${ }^{\mathrm{i}}$ PT: Positive thinking. 


\section{Discussion}

This study presents evidence indicating how coping patterns and mental health outcomes differed across various geographical regions during the early phase of COVID-19 pandemic. Our multiple group SEM analysis highlights the role of psychological flexibility as the only significant factor that mediated the relationship between illness perceptions toward COVID-19 and mental health across all included geographical regions. Indeed, several recent studies conducted in Italy [37], the United Kingdom [38,39], the United States [40-42], and Poland [43] have indicated how the facets of psychological flexibility play an important role in mitigating the impacts of the COVID-19 pandemic on mental health $[37-41,43,44]$. Of the aforementioned studies, some have further shown that the opposite processes of psychological flexibility, that is, the psychological inflexibility or experiential avoidance were positively associated with parenting stress and family discord [44], and moderated the suicidal risk in the context of COVID-19 stressors such as resource constraints and loss arising from the pandemic [42]. In literature, psychological flexibility has been regarded as a typical model of clinical psychological treatment, comprising the psychological processes related to acceptance, mindfulness and committed actions based on values $[16,32,45]$. Meta-analyses of clinical trials have highlighted the positive impacts of fostering psychological flexibility on mental health in clinical population groups such as diabetes [46], cancer [47], depression [48], anxiety spectrum disorders [48] and non-clinical population groups [49].

By conducting multi-group analyses, we found that despite differences in pandemic situations, social and health care contexts of the geographical regions, psychological flexibility remained as the only robust resilience factor against the adverse mental health impacts arising from COVID-19. Notably, in our study, we found that avoidance also showed a significant mediating role between illness perceptions toward COVID-19 and mental health, but it should not be considered as simply the inverse of psychological flexibility. As suggested by Dawson et al. [38], avoidance behaviors are natural human responses to an unknown threat, which can be adaptive in certain contexts (e.g., taking a short break from the sheer volume of COVID-19 related news that creates emotional disturbance [50]), but it could be a manifestation of psychological inflexibility if an individual fully engages in avoidance [38]. To summarize, our findings denote the importance of identifying, developing and evaluating a "trans-diagnostic" approach, that is, a psychotherapeutic intervention adopting the principles of acceptance, mindfulness and/or self-compassion to foster psychological flexibility for managing diverse mental health issues arising from the COVID-19 pandemic [51].

The significant mediating effect of seeking social support in the relationship between illness perceptions toward COVID-19 and mental health across most of the studied regions is consistent with recent evidence, supporting that increased social support has been found to protect individuals from developing mental health problems [52-54]. Social support refers to a series of support measures accessible to an individual through the social relationships with individuals, groups or the larger communities [55]. In literature, the benefits of social support on protecting individuals from developing mental health problems under COVID-19 has been illustrated [56,57]. Notably, the mediating role of seeking social support was not found in the participants from Eastern Asia region, which was those from Hong Kong. This result can be explained by the norm that Hong Kong people who most likely grew up under Eastern Asian culture, are less willing to seek explicit social support for dealing with stressful events [58]. If our sample could include participants from Mainland China so as to increase the representativeness of our samples under the Eastern Asia region, we might have been able to better examine whether social support could play a potential 'protecting' role in the detrimental mental health impacts of COVID-19 across both Western and Asian countries. In addition, no significant association was found between seeking social support and mental health in the Northern and Southern America sample, possibility due to the high levels of COVID-19 restrictions and lockdowns (as indicated by the highest mean score of the OxCGRT Indicator when compared to 
that of other regions) during the survey period, meaning people might have encountered difficulties in seeking direct social support from their communities.

The mediating role of prosociality as hypothesized in this study was only partially supported, as such relationship was only found in the Hong Kong and the Eastern Europe samples. In literature, studies have indicated that engaging in various forms of prosocial behaviors (i.e., helping for the benefits of others) would promote emotional well-being, empathy and social connectedness [59-61], while such positive impacts could be brought by mechanisms through influencing oxytocin release and reward circuitry system in the brain [61-63]. Furthermore, transcending self-interest to advance the welfare of others becomes an intrinsic motivation for adhering to public health measures against the COVID19 spread (e.g., physical distancing measures, wearing a face mask, social isolation rules to protect others from COVID-19, more than that of protecting oneself) [64], or to get vaccinated against COVID-19 $[65,66]$. It appears that prosociality has not yet been studied and compared across multi-regional samples in the COVID-19 context, as well as outbreaks of other novel infectious diseases and disasters. This implies the need for further cross-country longitudinal studies to better understand the inter-relationships between prosociality and mental health, together with other known psychosocial and environmental factors of the pandemic.

The mediating role of problem-solving was not found in all studied regions, except Northern Europe. Problem-solving is one of the adaptive coping strategies focusing on adapting practical steps to eliminate stress factors or reducing their impacts [67]. However, the evidence regarding whether problem-solving significantly correlates with mental health outcomes under the context of the COVID-19 pandemic remains mixed [30,68-70]. The non-significant result could be explained by the uncontrollable spread of the potentially fatal COVID-19, the pandemic context in which people are vulnerable to loneliness, and no effective treatments and vaccines were available at the time of survey implementation. Many people might be triggered by a sense of insecurity and inadequacy, which could be a potential stressor, and went beyond the use of problem-solving as a coping strategy to manage their psychological difficulties.

This study had limitations. Since the online survey was administrated during the early phase of the COVID-19 pandemic (April to June 2020) and the majority of the participating countries were in partial or complete lockdown, we relied on convenience sampling in which participant recruitment was mainly carried out through social networks and various media platforms online. Hence, the representativeness of the sample has been heavily skewed to adults in European countries (i.e., 68\% of the total sample). As Hong Kong was the only city out of other Eastern Asian countries or regions which participated in the survey, our findings may have limited generalizability to other Eastern Asian countries and other non-Western regions. Our convenience sampling method might not be able to reach those COVID-19 patients who had been hospitalized or are under treatment and we solely relied on self-reports, hence social desirability and response bias should be taken into account. In addition, when constructing and testing the mediational roles of coping, prosociality and psychological flexibility accounting for the relationship between illness perceptions toward COVID-19 and mental health, we followed the theoretical bases derived from the Common Sense Model of Self-Regulation for selecting and analyzing latent variables as predictors, mediators and outcomes, hence using cross-sectional data may mean we are unable to draw robust conclusions regarding the directionality of the aforementioned constructs. In each studied region, the variance to mental health contributed by psychological flexibility, prosociality and various significant coping factors ranged from $56 \%$ to $73 \%$, but there could be other explanatory variables, such as other coping factors and self-regulatory resources, which had been missed in our study. Even though our model adjusted for sociodemographic variables and the OxCGRT indicators, the possibility of other contextual factors affecting one's mental health, such as race, ethnicity, COVID-19 related morbidity and mortality outcomes, as well as social welfare systems across countries, cannot be ruled out. 


\section{Conclusions}

This large-scale cross-sectional survey examined how psychological flexibility, prosociality and coping mediated the impact of illness perceptions toward COVID-19 on mental health across seven geographical regions during the early phase of the pandemic. The findings pave important ways for the development of mental health interventions in navigating the current global health crisis. It is not surprising to see that people from different countries and regions exhibited different coping patterns, but they all shared the common ground in which fostering psychological flexibility played a key role in strengthening resilience. Perhaps, to support people across the globe in adapting to forthcoming COVID-19 or post-COVID-19 situational challenges, our primary health care efforts should shift to focus on fostering psychological flexibility, whether in addressing mental health needs as they arise within an individual, equipping groups (e.g., health care professionals) with skills that may foster resilience, or promoting psychological health in the broader population. One of the strategic goals determined by the World Health Organization (WHO) Special Initiative for Mental Health is the aim to increase quality and affordable community-based mental health care services for 100 million more people by 2023, so as to reduce health inequalities [71]. In addition, a recent report which summarized international experiences in the mental health response to COVID-19 has found that telehealth may soon become a core component in mental health services [72]. Hence, to maximize the reach of the psychotherapeutic interventions targeting psychological flexibility, various remote formats, such as social media platforms, mobile applications, or videoconferencing, should be adopted.

Author Contributions: Y.Y.C., A.T.G., M.K. and A.P.K. conceived the study. Y.Y.C. wrote the first draft of the manuscript. The statistical analyses were performed by Y.Y.C. All authors contributed to the data collection, data analysis, interpretation of results and critical revision of the manuscript. All authors have read and agreed to the published version of the manuscript.

Funding: The study was supported by the Seeding Research Fund, The Nethersole School of Nursing, Faculty of Medicine, The Chinese University of Hong Kong.

Institutional Review Board Statement: The ethics approval of the study was obtained from the Cyprus National Bioethics Committee (ЕЕВK ЕП 2020.01.60). All the participating countries in the COVID-19 IMPACT study obtained ethics approval from their corresponding ethic committees of the universities or governmental institutions and followed a standard protocol to conduct sampling, surveying, and data management. All procedures performed in this study involving human participants were in accordance with the 1964 Helsinki declaration and its later amendment.

Informed Consent Statement: Informed consent was obtained from all subjects involved in the study.

Data Availability Statement: The datasets used and/or analyzed during the current study are available from the corresponding author on reasonable request.

Acknowledgments: We would like to acknowledge the support from all the study participants, who donated their valuable time within the crisis period of the COVID-19 pandemic. We also thank Kaichow Choi, Senior Research Fellow and Statistician from The Nethersole Nursing of Nursing, Faculty of Medicine, The Chinese University of Hong Kong, for his expert advice on statistical analysis.

Conflicts of Interest: The authors declare no conflict of interest.

\section{References}

1. Arora, T.; Grey, I.; Östlundh, L.; Lam, K.B.H.; Omar, O.M.; Arnone, D. The prevalence of psychological consequences of COVID-19: A systematic review and meta-analysis of observational studies. J. Health Psychol. 2020, 1-20. [CrossRef] [PubMed]

2. Wu, T.; Jia, X.; Shi, H.; Niu, J.; Yin, X.; Xie, J.; Wang, X. Prevalence of mental health problems during the COVID-19 pandemic: A systematic review and meta-analysis. J. Affect. Disord. 2021, 281, 91-98. [CrossRef] [PubMed]

3. Asmundson, G.J.G.; Paluszek, M.M.; Landry, C.A.; Rachor, G.S.; McKay, D.; Taylor, S. Do pre-existing anxiety-related and mood disorders differentially impact COVID-19 stress responses and coping? J. Anxiety Disord. 2020, 74, 102271. [CrossRef] [PubMed] 
4. Krishnamoorthy, Y.; Nagarajan, R.; Saya, G.K.; Menon, V. Prevalence of psychological morbidities among general population, healthcare workers and COVID-19 patients amidst the COVID-19 pandemic: A systematic review and meta-analysis. Psychiatry Res. 2020, 293, 113382. [CrossRef]

5. Cénat, J.M.; Blais-Rochette, C.; Kokou-Kpolou, C.K.; Noorishad, P.-G.; Mukunzi, J.N.; McIntee, S.-E.; Dalexis, R.D.; Goulet, M.-A.; Labelle, P.R. Prevalence of symptoms of depression, anxiety, insomnia, posttraumatic stress disorder, and psychological distress among populations affected by the COVID-19 pandemic: A systematic review and meta-analysis. Psychiatry Res. 2021, 295, 113599. [CrossRef]

6. Salari, N.; Khazaie, H.; Hosseinian-Far, A.; Khaledi-Paveh, B.; Kazeminia, M.; Mohammadi, M.; Shohaimi, S.; Daneshkhah, A.; Eskandari, S. The prevalence of stress, anxiety and depression within front-line healthcare workers caring for COVID-19 patients: A systematic review and meta-regression. Hum. Resour. Health 2020, 18, 100. [CrossRef]

7. Muller, A.E.; Hafstad, E.V.; Himmels, J.P.W.; Smedslund, G.; Flottorp, S.; Stensland, S.Ø.; Stroobants, S.; Van de Velde, S.; Vist, G.E. The mental health impact of the Covid-19 pandemic on healthcare workers, and interventions to help them: A rapid systematic review. Psychiatry Res. 2020, 293, 113441. [CrossRef]

8. Huberfeld, N.; Gordon, S.H.; Jones, D.K. Federalism complicates the response to the COVID-19 health and economic crisis: What can be done? J. Health Polit. Policy Law 2020, 45, 951-965. [CrossRef]

9. Gordon, S.H.; Huberfeld, N.; Jones, D.K. What federalism means for the US response to coronavirus disease 2019. JAMA Health Forum 2020, 1, e200510. [CrossRef]

10. Desson, Z.; Lambertz, L.; Peters, J.W.; Falkenbach, M.; Kauer, L. Europe's Covid-19 outliers: German, Austrian and Swiss policy responses during the early stages of the 2020 pandemic. Health Policy Technol. 2020, 9, 405-418. [CrossRef]

11. Han, E.; Tan, M.M.J.; Turk, E.; Sridhar, D.; Leung, G.M.; Shibuya, K.; Asgari, N.; Oh, J.; García-Basteiro, A.L.; Hanefeld, J.; et al. Lessons learnt from easing COVID-19 restrictions: An analysis of countries and regions in Asia Pacific and Europe. Lancet 2020, 396, 1525-1534. [CrossRef]

12. Tso, R.V.; Cowling, B.J. Importance of Face Masks for COVID-19: A call for effective public education. Clin. Infec. Dis. 2020, 71, 2195-2198. [CrossRef]

13. Allel, K.; Tapia-Muñoz, T.; Morris, W. Country-level factors associated with the early spread of COVID-19 cases at 5, 10 and 15 days since the onset. Glob. Public Health 2020, 15, 1589-1602. [CrossRef]

14. Hagger, M.S.; Orbell, S. The common sense model of illness self-regulation: A conceptual review and proposed extended model. Health Psychol. Rev. 2021, 1-31. [CrossRef]

15. Hagger, M.S.; Koch, S.; Chatzisarantis, N.L.D.; Orbell, S. The common sense model of self-regulation: Meta-analysis and test of a process model. Psychol. Bull. 2017, 143, 1117-1154. [CrossRef]

16. Kashdan, T.B.; Rottenberg, J. Psychological flexibility as a fundamental aspect of health. Clin. Psychol. Rev. 2010, 30, 865-878. [CrossRef]

17. Caprara, G.V.; Alessandri, G.; Eisenberg, N. Prosociality: The contribution of traits, values, and self-efficacy beliefs. J. Pers. Soc. Psychol. 2012, 102, 1289-1303. [CrossRef]

18. Chong, Y.Y.; Chien, W.; Cheng, H.Y.; Kassianos, A.; Gloster, A.; Karekla, M. Can psychological flexibility and prosociality mitigate illness perceptions toward COVID-19 on mental health? A cross-sectional study among Hong Kong adults. Glob. Health 2021, 17, 43. [CrossRef]

19. Adamson, M.M.; Phillips, A.; Seenivasan, S.; Martinez, J.; Grewal, H.; Kang, X.; Coetzee, J.; Luttenbacher, I.; Jester, A.; Harris, O.A.; et al. International prevalence and correlates of psychological stress during the global COVID-19 pandemic. Int. J. Environ. Res. Public Health 2020, 17, 9248. [CrossRef]

20. Faulkner, J.; O’Brien, W.J.; McGrane, B.; Wadsworth, D.; Batten, J.; Askew, C.D.; Badenhorst, C.; Byrd, E.; Coulter, M.; Draper, N.; et al. Physical activity, mental health and well-being of adults during initial COVID-19 containment strategies: A multi-country cross-sectional analysis. J. Sci. Med. Sport 2021, 24, 320-326. [CrossRef]

21. Généreux, M.; Schluter, P.J.; Hung, K.K.C.; Wong, C.S.; Mok, C.P.Y.; O’sullivan, T.; David, M.D.; Carignan, M.-E.; Blouin-Genest, G.; Champagne-Poirier, O.; et al. One virus, four continents, eight countries: An interdisciplinary and international study on the psychosocial impacts of the covid-19 pandemic among adults. Int. J. Environ. Res. Public Health 2020, 17, 8390. [CrossRef] [PubMed]

22. Bartoszek, A.; Walkowiak, D.; Bartoszek, A.; Kardas, G. Mental well-being (depression, loneliness, insomnia, daily life fatigue) during COVID-19 related home-confinement-A study from Poland. Int. J. Environ. Res. Public Health 2020, 17, 7417. [CrossRef] [PubMed]

23. Gloster, A.T.; Lamnisos, D.; Lubenko, J.; Presti, G.; Squatrito, V.; Constantinou, M.; Nicolaou, C.; Papacostas, S.; Aydin, G.; Chong, Y.Y.; et al. Impact of COVID-19 pandemic on mental health: An international study. PLoS ONE 2020, 15, e0244809. [CrossRef] [PubMed]

24. Chong, Y.Y.; Chien, W.T.; Cheng, H.Y.; Chow, K.M.; Kassianos, A.P.; Karekla, M.; Gloster, A. The role of illness perceptions, coping, and self-efficacy on adherence to precautionary measures for COVID-19. Int. J. Environ. Res. Public Health 2020, 17, 6540. [CrossRef] [PubMed]

25. Lamers, S.M.A.; Westerhof, G.J.; Bohlmeijer, E.T.; Ten Klooster, P.M.; Keyes, C.L.M. Evaluating the psychometric properties of the Mental Health Continuum-Short Form (MHC-SF). J. Clin. Psychol. 2011, 67, 99-110. [CrossRef] 
26. Guo, C.; Tomson, G.; Guo, J.; Li, X.; Keller, C.; Söderqvist, F. Psychometric evaluation of the Mental Health Continuum-Short Form (MHC-SF) in Chinese adolescents-A methodological study. Health Qual. Life Outcomes 2015, 13, 198. [CrossRef] [PubMed]

27. Broadbent, E.; Wilkes, C.; Koschwanez, H.; Weinman, J.; Norton, S.; Petrie, K.J. A systematic review and meta-analysis of the Brief Illness Perception Questionnaire. Health Psychol. 2015, 30, 1361-1385. [CrossRef]

28. Rosenstock, I.M.; Strecher, V.J.; Becker, M.H. Social learning theory and the health belief model. Health Educ. Q. 1988, 15, 175-183. [CrossRef]

29. Baumstarck, K.; Alessandrini, M.; Hamidou, Z.; Auquier, P.; Leroy, T.; Boyer, L. Assessment of coping: A new French four-factor structure of the brief COPE inventory. Health Qual. Life Outcomes 2017, 15, 8. [CrossRef]

30. Chew, Q.H.; Chia, F.L.-A.; Ng, W.K.; Lee, W.C.I.; Tan, P.L.L.; Wong, C.S.; Puah, S.H.; Shelat, V.G.; Seah, E.-J.D.; Huey, C.W.T.; et al. Perceived stress, stigma, traumatic stress levels and coping responses amongst residents in training across multiple specialties during COVID-19 pandemic-A longitudinal study. Int. J. Environ. Res. Public Health 2020, 17, 6572. [CrossRef]

31. Marguerite, S.; Laurent, B.; Marine, A.; Tanguy, L.; Karine, B.; Pascal, A.; Xavier, Z. Actor-partner interdependence analysis in depressed patient-caregiver dyads: Influence of emotional intelligence and coping strategies on anxiety and depression. Psychiatry Res. 2017, 258, 396-401. [CrossRef] [PubMed]

32. Hayes, S.C. Acceptance and Commitment Therapy: The Process and Practice of Mindful Change, 2nd ed.; Guilford Press: New York, NY, USA, 2012.

33. Villanueva, J.; Meyer, A.H.; Rinner, M.T.B.; Firsching, V.J.; Benoy, C.; Brogli, S.; Walter, M.; Bader, K.; Gloster, A.T. “Choose change": Design and methods of an acceptance and commitment therapy effectiveness trial for transdiagnostic treatment-resistant patients. BMC Psychiatry 2019, 19, 173. [CrossRef] [PubMed]

34. Caprara, G.V.; Steca, P.; Zelli, A.; Capanna, C. A new scale for measuring adults' prosocialness. Eur. J. Psychol. Assess. 2005, 21, 77-89. [CrossRef]

35. Hale, T.; Petherick, A.; Phillips, T.; Webster, S. Variation in government responses to COVID-19. In Blavatnik School of Government Working Paper; University of Oxford: Oxford, UK, 2020; Volume 31, p. 2020-11.

36. United Nations, Department of Economic and Social Affairs, Population Division. World Population Prospects 2019, Volume II: Demographic Profiles (ST/ESA/SER.A/427). 2019. Available online: https://population.un.org/wpp/Publications/Files/WPP2 019_Volume-II-Demographic-Profiles.pdf (accessed on 20 March 2021).

37. Pakenham, K.I.; Landi, G.; Boccolini, G.; Furlani, A.; Grandi, S.; Tossani, E. The moderating roles of psychological flexibility and inflexibility on the mental health impacts of COVID-19 pandemic and lockdown in Italy. J. Contextual Behav. Sci. 2020, 17, 109-118. [CrossRef]

38. Dawson, D.L.; Golijani-Moghaddam, N. COVID-19: Psychological flexibility, coping, mental health, and wellbeing in the UK during the pandemic. J. Contextual Behav. Sci. 2020, 17, 126-134. [CrossRef]

39. McCracken, L.M.; Badinlou, F.; Buhrman, M.; Brocki, K.C. The role of psychological flexibility in the context of COVID-19: Associations with depression, anxiety, and insomnia. J. Contextual Behav. Sci. 2021, 19, 28-35. [CrossRef]

40. Kroska, E.B.; Roche, A.I.; Adamowicz, J.L.; Stegall, M.S. Psychological flexibility in the context of COVID-19 adversity: Associations with distress. J. Contextual Behav. Sci. 2020, 18, 28-33. [CrossRef]

41. Peltz, J.S.; Daks, J.S.; Rogge, R.D. Mediators of the association between COVID-19-related stressors and parents' psychological flexibility and inflexibility: The roles of perceived sleep quality and energy. J. Contextual Behav. Sci. 2020, 17, 168-176. [CrossRef]

42. Crasta, D.; Daks, J.S.; Rogge, R.D. Modeling suicide risk among parents during the COVID-19 pandemic: Psychological inflexibility exacerbates the impact of COVID-19 stressors on interpersonal risk factors for suicide. J. Contextual Behav. Sci. 2020, 18, 117-127. [CrossRef]

43. Wielgus, B.; Urban, W.; Patriak, A.; Cichocki, Ł. Examining the associations between psychological flexibility, mindfulness, psychosomatic functioning, and anxiety during the COVID-19 pandemic: A path analysis. Int. J. Environ. Res. Public Health 2020, 17, 8764. [CrossRef]

44. Daks, J.S.; Peltz, J.S.; Rogge, R.D. Psychological flexibility and inflexibility as sources of resiliency and risk during a pandemic: Modeling the cascade of COVID-19 stress on family systems with a contextual behavioral science lens. J. Contextual Behav. Sci. 2020, 18, 16-27. [CrossRef]

45. Gloster, A.T.; Karekla, M. A multi-level, multi-method approach to testing and refining intervention targets. In Beyond the DSM; Hayes, S., Hofmann, S., Eds.; New Harbinger: Oakland, CA, USA, 2020.

46. Ngan, H.Y.; Chong, Y.Y.; Chien, W.T. Effects of mindfulness- and acceptance-based interventions on diabetes distress and glycaemic level in people with type 2 diabetes: Systematic review and meta-analysis. Diabet. Med. 2021, e14525. [CrossRef]

47. Li, H.; Wong, C.L.; Jin, X.; Chen, J.; Chong, Y.Y.; Bai, Y. Effects of Acceptance and Commitment Therapy on health-related outcomes for patients with advanced cancer: A systematic review. Int. J. Nurs. Stud. 2021, 115, 103876. [CrossRef]

48. Gloster, A.T.; Walder, N.; Levin, M.E.; Twohig, M.P.; Karekla, M. The empirical status of acceptance and commitment therapy: A review of meta-analyses. J. Contextual Behav. Sci. 2020, 18, 181-192. [CrossRef]

49. French, K.; Golijani-Moghaddam, N.; Schröder, T. What is the evidence for the efficacy of self-help acceptance and commitment therapy? A systematic review and meta-analysis. J. Contextual Behav. Sci. 2017, 6, 360-374. [CrossRef]

50. Chong, Y.Y.; Cheng, H.Y.; Chan, H.Y.L.; Chien, W.T.; Wong, S.Y.S. COVID-19 pandemic, infodemic and the role of eHealth literacy. Int. J. Nurs. Stud. 2020, 108, 103644. [CrossRef] 
51. Biglan, A.; Johansson, M.; Van Ryzin, M.; Embry, D. Scaling up and scaling out: Consilience and the evolution of more nurturing societies. Clin. Psychol. Rev. 2020, 81, 101893. [CrossRef]

52. Grey, I.; Arora, T.; Thomas, J.; Saneh, A.; Tohme, P.; Abi-Habib, R. The role of perceived social support on depression and sleep during the COVID-19 pandemic. Psychiatry Res. 2020, 293, 113452. [CrossRef]

53. Ye, Z.; Yang, X.; Zeng, C.; Wang, Y.; Shen, Z.; Li, X.; Lin, D. Resilience, social support, and coping as mediators between COVID19-related stressful experiences and acute stress disorder among college students in China. Appl. Psychol. Health Well-Being 2020 12, 1074-1094. [CrossRef]

54. Saltzman, L.Y.; Hansel, T.C.; Bordnick, P.S. Loneliness, isolation, and social support factors in post-COVID-19 mental health. Psychol. Trauma 2020, 12, S55-S57. [CrossRef]

55. Taylor, S.E. Social support: A review. In The Oxford Handbook of Health Psychology; Friedman, H.S., Ed.; Oxford University Press: Oxford, UK; New York, NY, USA, 2011.

56. Bonavita, S.; Sparaco, M.; Russo, A.; Borriello, G.; Lavorgna, L. Perceived stress and social support in a large population of people with multiple sclerosis recruited online through the COVID-19 pandemic. Eur. J. Neurol. 2021. [CrossRef] [PubMed]

57. Li, F.; Luo, S.; Mu, W.; Li, Y.; Ye, L.; Zheng, X.; Xu, B.; Ding, Y.; Ling, P.; Zhou, M.; et al. Effects of sources of social support and resilience on the mental health of different age groups during the COVID-19 pandemic. BMC Psychiatry 2021, 21, 16. [CrossRef] [PubMed]

58. Chang, J.; Chen, C.-N.; Alegría, M. Contextualizing social support: Pathways to help seeking in Latinos, Asian Americans, and whites. J. Soc. Clin. Psychol. 2014, 33, 1-24. [CrossRef]

59. Gloster, A.T.; Rinner, M.T.B.; Meyer, A.H. Increasing prosocial behavior and decreasing selfishness in the lab and everyday life. Sci. Rep. 2020, 10, 21220. [CrossRef]

60. Laguna, M.; Mazur, Z.; Kędra, M.; Ostrowski, K. Interventions stimulating prosocial helping behavior: A systematic review. J. Appl. Soc. Psychol. 2020, 50, 676-696. [CrossRef]

61. Raposa, E.B.; Laws, H.B.; Ansell, E.B. Prosocial behavior mitigates the negative effects of stress in everyday life. Clin. Psychol. Sci. 2016, 4, 691-698. [CrossRef]

62. Preston, S.D. The origins of altruism in offspring care. Psychol. Bull. 2013, 139, 1305-1341. [CrossRef]

63. Gilbert, P. Affiliative and prosocial motives and emotions in mental health. Dialogues Clin. Neurosci. 2015, 17, 381-389.

64. Heffner, J.; Vives, M.-L.; FeldmanHall, O. Emotional responses to prosocial messages increase willingness to self-isolate during the COVID-19 pandemic. Personal. Individ. Differ. 2021, 170, 110420. [CrossRef]

65. Szmyd, B.; Bartoszek, A.; Karuga, F.F.; Staniecka, K.; Błaszczyk, M.; Radek, M. Medical students and SARS-CoV-2 vaccination: Attitude and behaviors. Vaccines 2021, 9, 128. [CrossRef]

66. Szmyd, B.; Karuga, F.F.; Bartoszek, A.; Staniecka, K.; Siwecka, N.; Bartoszek, A.; Błaszczyk, M.; Radek, M. Attitude and behaviors towards SARS-CoV-2 vaccination among healthcare workers: A cross-sectional study from Poland. Vaccines 2021, 9, 218. [CrossRef]

67. Skinner, E.A.; Edge, K.; Altman, J.; Sherwood, H. Searching for the structure of coping: A review and critique of category systems for classifying ways of coping. Psychol. Bull. 2003, 129, 216-269. [CrossRef]

68. Babore, A.; Lombardi, L.; Viceconti, M.L.; Pignataro, S.; Marino, V.; Crudele, M.; Candelori, C.; Bramanti, S.M.; Trumello, C. Psychological effects of the COVID-2019 pandemic: Perceived stress and coping strategies among healthcare professionals. Psychiatry Res. 2020, 293, 113366. [CrossRef]

69. Agha, S. Mental well-being and association of the four factors coping structure model: A perspective of people living in lockdown during COVID-19. Ethics Med. Public Health 2021, 16, 100605. [CrossRef]

70. Korkmaz, S.; Kazgan, A.; Çekiç, S.; Tartar, A.S.; Balcı, H.N.; Atmaca, M. The anxiety levels, quality of sleep and life and problem-solving skills in healthcare workers employed in COVID-19 services. J. Clin. Neurosci. 2020, 80, 131-136. [CrossRef]

71. World Health Organization. The WHO Special Initiative for Mental Health (2019-2023): Universal Health Coverage for Mental Health. Available online: https://apps.who.int/iris/bitstream/handle/10665/310981/WHO-MSD-19.1-eng.pdf?sequence= $1 \&$ isAllowed=y (accessed on 4 April 2021).

72. Rosenberg, S.; Mendoza, J.; Tabatabaei-Jafari, H.; Salvador-Carulla, L. International experiences of the active period of COVID19-Mental health care. Health Policy Technol. 2020, 9, 503-509. [CrossRef] 